\title{
Mesh adaptivity in optimal control of elliptic variational inequalities with point-tracking of the state
}

\author{
ChARLES BRETT \\ MASDOC CDT, Mathematics Institute, \\ University of Warwick, Coventry, CV4 7AL, U \\ E-mail: c.brett@warwick.ac.uk \\ Charles M. ElliotT \\ Mathematics Institute, \\ University of Warwick, Coventry CV4 7AL, UK \\ E-mail:c.m.elliott@warwick.ac.uk \\ MiCHAEL HINTERMÜLLER \\ Department of Mathematics, Humboldt-Universität zu Berlin, \\ Unter den Linden 6, 10099 Berlin, Germany \\ E-mail:hint@math.hu-berlin.de \\ CAROLINE LÖBHARD \\ Department of Mathematics, Humboldt-Universität zu Berlin, \\ Unter den Linden 6, 10099 Berlin, Germany \\ E-mail:loebhard@math.hu-berlin.de
}

[Received 8 November 2013 and in revised form 29 November 2014]

\begin{abstract}
An adaptive finite element method is developed for a class of optimal control problems with elliptic variational inequality constraints and objective functionals defined on the space of continuous functions, necessitated by a point-tracking requirement with respect to the state variable. A suitable first order stationarity concept is derived for the problem class via a penalty technique. The dualweighted residual approach for goal-oriented adaptive finite elements is applied and relies on the stationarity system. It yields primal residuals weighted by approximate dual quantities and vice versa as well as complementarity mismatch errors. A report on numerical tests, including the critical case of biactivity, completes this work.
\end{abstract}

2010 Mathematics Subject Classification: Primary 49K20, 65K15, 90C33.

Keywords: Adaptive finite element methods, optimal control of variational inequalities in function space, point-tracking, C-stationarity, goal-oriented error estimation.

\section{Introduction}

In this paper we study a class of optimal control problems with variational inequality and pointwise control constraints, as well as an objective containing the tracking of the state variable at specific points. Such models are of interest, for example, in inverse problems where a parameter has to be identified from measurements at predefined locations in the underlying domain; see [1] for an application in mathematical finance. 
Since variational inequalities often model an equilibrium condition, the problems treated here are called mathematical programs with equilibrium constraints (MPECs) in the literature. This problem class has been studied since the 1970s in finite dimensional spaces and later also in function space; see [38, 43] and [6, 41] for extensive accounts of the progress in the respective subjects. Despite the research efforts ever since, the problem class considered in this paper has not yet been analyzed. The degeneracy of the constraint set which is typical for MPECs makes standard methods for the derivation of necessary optimality conditions inapplicable. As an alternative, penalty methods (see, e.g., [6] and the references therein) and methods relying on generalized differentiability concepts (see, e.g., [40]) have been used. Concerning the state system of the present work, useful analytic properties (such as stability and directional differentiability) of solution operators of variational inequalities are discussed in [15, 34, 45]. These properties were employed successfully to derive alternative forms of stationarity. For such concepts in function space we refer to [28, 42], and to [47] for finite dimensional problems.

Besides their value for the design of mesh independent solution algorithms, function space stationarity conditions can be employed to derive goal oriented a posteriori error indicators with respect to the objective functional in the context of finite element discretizations. In optimization with partial differential equation constraints, this concept was pioneered in $[4,10]$, and an associated adaptive method for an MPEC is given in [27]. The method relies on local error indicators for the implementation of an automatic adaption of the discrete space. For different approaches to adaptive finite element methods (AFEMs) we refer to the monographs [3, 4, 44, 49] and the references therein. The typical dual-weighted residual error estimators have been successfully applied to optimal control problems with partial differential constraints e.g. in [11, 23-26, 37, 46, 50]. Alternatively, functional error estimation for optimal control problems is analyzed in [20], and residual based estimators are studied in [19, 30-33, 36, 37]. In particular, a residual based estimator for the optimal control of an obstacle problem is suggested in [19].

In the present paper, we analyze the new problem class and derive suitable stationarity conditions with a smooth penalty approach and an averaging technique for the function evaluations in the objective. Some of the analytic complications associated with the underlying problem class are due to the point tracking which requires a state space which embeds into continuous functions, and thus leads to reduced regularity of adjoint states. Based on the resulting so-called $\epsilon$-almost Cstationarity system, we develop a goal-oriented indicator for the error in the objective functional of the optimal control problem which contains dual weighted residuals and terms covering the mismatch in complementarity of primal variables and the respective multipliers. These are similar to the ones in [27], but with extra terms concerning the supplementary control constraints.

The paper is structured as follows: We provide a collection of basic notation in the rest of this section. In Section 2 we state the considered MPEC and prove a regularity result for the solution of the variational inequality. In addition, the penalization scheme with a new averaging technique for the objective is defined. Section 3 shows the limiting process that takes the first order optimality conditions of the auxiliary problem to the $\epsilon$-almost C-stationarity conditions for the MPEC. These conditions are employed for the derivation of an abstract error representation formula and the a posteriori error indicators in Section 4. We finish in Section 5 with a brief description of the solution method and document numerical results.

Notation. For a Banach space $Y$ and its dual $Y^{*}$, the dual pairing of elements $y^{*} \in Y^{*}$ and $y \in Y$ is written as $\left\langle y^{*}, y\right\rangle_{Y^{*}}=y^{*}(y)$. In a reflexive Banach space $Y$ with the identification $i: Y \rightarrow Y^{* *}$, we write $\left\langle y, y^{*}\right\rangle_{Y}:=\left\langle i(y), y^{*}\right\rangle_{Y^{* *}}$. If $X$ is a Hilbert space, we write $\left(x_{1}, x_{2}\right)_{X}$ 
for the scalar product of $x_{1}, x_{2} \in X$. For sequences $\left(y_{k}\right)_{k \in \mathbb{N}} \subset Y$ and $\left(y_{k}^{*}\right)_{k \in \mathbb{N}} \subset Y^{*}$, we denote the strong convergence to a limit $y \in Y$ (i.e., $\left\|y_{k}-y\right\|_{Y} \rightarrow 0$ for $k \rightarrow \infty$ ) by $y_{k} \rightarrow y$, whereas weak and weak* convergence are denoted by $y_{k} \rightarrow y$ (i.e., $\forall z^{*} \in Y^{*},\left\langle z^{*}, y_{k}-y\right\rangle_{Y^{*}} \rightarrow 0$ ) and $y_{k}^{*} \rightarrow^{*} y^{*}$ (i.e., $\forall z \in Y,\left\langle y_{k}^{*}-y^{*}, z\right\rangle_{Y^{*}} \rightarrow 0$ ).

The standard Lebesgue and Sobolev spaces on an open, bounded Lipschitz domain $\Omega$ are denoted in the usual way and we use the norms $\|v\|_{H_{0}^{1}(\Omega)}:=\|\nabla v\|_{L^{2}(\Omega)}$ and $\|v\|_{W_{0}^{1, q}(\Omega)}:=$ $\|\nabla v\|_{L^{q}(\Omega)}$. Furthermore we write $C^{0}(\bar{\Omega})$ for continuous functions on the closure of $\Omega$ and identify its dual space $\left(C^{0}(\bar{\Omega})\right)^{*}$ with the space of bounded Borel measures $m_{b}(\bar{\Omega})$ by the RieszAlexandroff representation theorem.

For a function $\psi \in L^{2}(\Omega)$, we define $(\psi)_{+}=\max (0, \psi)$ in a pointwise almost everywhere (a.e.) sense. The characteristic function of a subset $M \subset \Omega$ is denoted by $\chi_{M}: \Omega \rightarrow\{0,1\}$, while $|M|$ is the Lebesgue measure of $M$ provided $M$ is measurable. We write $\mathbb{R}^{>0}=\{t \in \mathbb{R} \mid t>0\}$ and, for a subset $M$ of a Banach space $Y, M^{+}=\left\{y^{*} \in Y^{*} \mid \forall y \in M: y^{*}(y) \geqslant 0\right\} \subset Y^{*}$. The number of elements in a finite set $I$ is denoted by $\operatorname{card}(I)$.

Throughout the text, $C>0$ is a generic constant which depends only on the problem input data, but not on solutions, or on the discretization.

\section{Model problem and the penalization scheme}

\subsection{Statement of the problem}

For an open bounded domain $\Omega \subset \mathbb{R}^{2}$, we consider $a_{i j} \in L^{\infty}(\Omega)(i, j \in\{1,2\})$ such that for all $\zeta=\left(\zeta_{1}, \zeta_{2}\right) \in \mathbb{R}^{2}$ and almost all $x \in \Omega$,

$$
\sum_{i, j=1}^{2} a_{i j}(x) \zeta_{i} \zeta_{j} \geqslant \alpha|\zeta|^{2},
$$

where $\alpha>0$, and we define the uniformly coercive operator $A: H_{0}^{1}(\Omega) \rightarrow H^{-1}(\Omega)$ by

$$
A=-\sum_{i, j=1}^{2} \frac{\partial}{\partial x_{i}} a_{i j} \frac{\partial}{\partial x_{j}}=-\operatorname{div}\left(\left(a_{i j}\right) \nabla\right) .
$$

Here and below, the coefficient matrix in $A$ is denoted by $\left(a_{i j}\right) \in L^{\infty}(\Omega)^{2 \times 2}$. Let $V=H_{0}^{1}(\Omega)$, $A: V \rightarrow V^{*}$ and $K=\{z \in V \mid z \geqslant 0$ a.e. in $\Omega\}$. By the Lions-Stampacchia theorem (cf. [45, $\mathrm{Thm} .4: 3.1])$, the variational inequality problem

$$
\text { Find } y \in K \text { such that }\langle A y, z-y\rangle_{H^{-1}(\Omega)} \geqslant\langle f+u, z-y\rangle_{H^{-1}(\Omega)} \quad \forall z \in K \text {, }
$$

has a unique solution for all $u, f \in V^{*}$. This solution can be equivalently characterized by the following complementarity system with slack variable $\xi$,

$$
\xi=A y-f-u \in V^{*}, \quad y \geqslant 0 \text { in } V, \quad \xi \geqslant 0 \text { in } V^{*}, \quad\langle\xi, y\rangle_{H^{-1}(\Omega)}=0 .
$$

In a slight misuse of notation, we denote the solution operator of (2.2) by $y(\cdot)$, so this maps $u \in V^{*}$ to $y=y(u)$. It is well-known from [13] (see also [45, Thm.5:2.5 (i)]) that for $\partial \Omega \in C^{1}, a_{i j} \in$ 
$L^{\infty}(\Omega) \forall i, j \in\{1,2\}$ and $f+u \in W^{-1, q}(\Omega)$, there exists $\hat{q}>2$ such that for all $2<q<\hat{q}$ the solution of (2.2) satisfies $y(u) \in W_{0}^{1, q}(\Omega)$. The following proposition shows that in fact, less regularity of the boundary $\partial \Omega$ is necessary to prove that the solution operator of (2.2) maps $L^{2}(\Omega)$ into $W_{0}^{1, q}(\Omega)$.

Proposition 2.1 Assume that $\Omega$ is of class $C^{0,1}$ (i.e. has a Lipschitz boundary, which includes non-convex polygonal domains), $a_{i j} \in L^{\infty}(\Omega) \forall i, j \in\{1,2\}$ and $f+u \in L^{2}(\Omega)$. Then there exists $Q>2$ such that for all $2<q<Q$ the solution of (2.2) satisfies $y(u) \in W_{0}^{1, q}(\Omega)$ and the following estimate holds,

$$
\|y(u)\|_{W_{0}^{1, q}(\Omega)} \leqslant C\|f+u\|_{W^{-1, q}(\Omega)} .
$$

Proof. In the same way as in [35, IV, Theorem 2.3], we define $\vartheta_{\tau}: \mathbb{R} \rightarrow[0,1]$ for $\tau>0$ as

$$
\vartheta_{\tau}(t)=\left\{\begin{array}{cl}
1, & t \leqslant 0, \\
1-\frac{t}{\tau}, & 0<t<\tau, \\
0, & t \geqslant \tau
\end{array}\right.
$$

and approximate the variational inequality by the problem

$$
\text { Find } \quad y \in H_{0}^{1}(\Omega) \quad \text { such that } \quad A y=\max (0,-f-u) \vartheta_{\tau}(y)+f+u \text {. }
$$

Lemma 2.2 in [35, IV] yields a unique solution $y_{\tau} \in H_{0}^{1}(\Omega)$. The Lipschitz domain $\Omega$ is regular in the sense of [22, Def. 2], and thus by [22, Thm. 3] there exists $\hat{q}>2$ such that the duality map $-\Delta: H_{0}^{1}(\Omega) \rightarrow H^{-1}(\Omega)$ maps $W_{0}^{1, \hat{q}}(\Omega)$ onto $W^{-1, \hat{q}}(\Omega)$ and $\|\phi\|_{W_{0}^{1, q}(\Omega)} \leqslant C\|-\Delta \phi\|_{W^{-1, q}(\Omega)}$ for all $\phi \in W_{0}^{1, q}(\Omega)$. So from Meyer's estimate (see [39, Thm.1]) we obtain $Q \in(2, \hat{q}]$, depending only on $\Omega$, the ellipticity constant and the norm of $\left(a_{i j}\right)$, such that $A$ maps $W_{0}^{1, q}(\Omega)$ onto $W^{-1, q}(\Omega)$ for all $2<q<Q$, and we have the respective norm estimate. The right hand side of (2.5) is in $W^{-1, q}(\Omega)$ so the solution satisfies $y_{\tau} \in W_{0}^{1, q}(\Omega)$ and

$$
\begin{aligned}
\left\|y_{\tau}\right\|_{W_{0}^{1, q}(\Omega)} & \leqslant C\left\|A y_{\tau}\right\|_{W^{-1, q}(\Omega)} \leqslant C\left\|\max (0,-f-u) \vartheta_{\tau}\left(y_{\tau}\right)+f+u\right\|_{W^{-1, q}(\Omega)} \\
& \leqslant C\|f+u\|_{W^{-1, q}(\Omega)} .
\end{aligned}
$$

Thus for a sequence $\tau_{k} \rightarrow 0,\left(y_{\tau_{k}}\right)_{k \in \mathbb{N}}$ is uniformly bounded in $W_{0}^{1, q}(\Omega)$ and therefore contains a weakly convergent subsequence with limit $\bar{y} \in W_{0}^{1, q}(\Omega)$. Following the arguments in [35], we find that the limit satisfies the variational inequality, i.e. $\bar{y}=y(u)$. The uniqueness of this solution implies the weak convergence of the full sequence. The weak lower semi-continuity of the norm implies

$$
\|y(u)\|_{W_{0}^{1, q}(\Omega)} \leqslant \liminf \left\{\left\|y_{\tau_{k}}\right\|_{W_{0}^{1, q}(\Omega)} \mid k \in \mathbb{N}\right\} \leqslant C\|f+u\|_{W^{-1, q}(\Omega)} .
$$

In the rest of this paper we assume that $\Omega$ is a Lipschitz domain in $\mathbb{R}^{2}$ and that $q \in(2, Q)$, with $Q>2$ from Proposition 2.1. In this way, $W_{0}^{1, q}(\Omega)$ embeds into $C(\bar{\Omega})$, and thus an optimal control problem with objective $J: C(\bar{\Omega}) \times L^{2}(\Omega) \rightarrow \mathbb{R}$ can be treated. Since $L^{2}(\Omega) \cong L^{2}(\Omega)^{*} \subset$ $W^{-1, q}(\Omega)$, we can consider the solution operator as a mapping $y: L^{2}(\Omega) \rightarrow W_{0}^{1, q}(\Omega)$. 
We analyze optimal control problems with point-tracking of the state variable $y$ in the objective, and with the constraint (2.2) as well as optional $L^{2}(\Omega)$-box-constraints on the control variable $u$, i.e.,

$$
\begin{aligned}
& \text { Minimize } \quad J(y, u)=\frac{1}{2} \sum_{w \in I}\left(y(w)-y_{w}\right)^{2}+\frac{v}{2}\|u\|_{L^{2}(\Omega)}^{2} \\
& \text { over } \\
& \text { subject to (s.t.) } \\
& (y, u) \in W_{0}^{1, q}(\Omega) \times L^{2}(\Omega), \\
& y=y(u) \text { solves }(2.2) \text {, } \\
& u \in U_{a d}=\left\{u \in L^{2}(\Omega) \mid a \leqslant u \leqslant b \text { a.e. in } \Omega\right\} \text {, }
\end{aligned}
$$

where $w$ denotes a tracking point in a finite set $I \subset \Omega, y_{w} \in \mathbb{R}$ is a desired (or measured) value of the state $y$ at $w, v>0$ is the cost of the control, $f \in L^{2}(\Omega)$, and the bounds $a, b \in L^{2}(\Omega)$ satisfy $a<b$ almost everywhere in $\Omega$, or $a=-\infty$ and $b=\infty$. The function evaluation of $y \in W_{0}^{1, q}(\Omega)$ in $w$ is also denoted by $\left\langle\delta_{w}, y\right\rangle_{W^{-1, q^{\prime}}(\Omega)}$, where $\delta_{w} \in W^{-1, q^{\prime}}(\Omega)=\left(W_{0}^{1, q}(\Omega)\right)^{*}$ with $q^{\prime}=\frac{q}{q-1}$. The objective functional $J: W_{0}^{1, q}(\Omega) \times L^{2}(\Omega) \rightarrow \mathbb{R}$ is continuous and convex, and thus weakly lower semi-continuous. In its reduced form, the control problem reads

$$
\text { Minimize } \hat{J}(u)=\frac{1}{2} \sum_{w \in I}\left|y(u)(w)-y_{w}\right|^{2}+\frac{v}{2}\|u\|_{L^{2}(\Omega)}^{2} \quad \text { over } u \in U_{a d}
$$

REMARK 2.2 The differential operator $A$ is a topological isomorphism from $W_{0}^{1, q}(\Omega)$ into $W^{-1, q}(\Omega)$ according to the proof of Proposition 2.1. We denote the adjoint of $A$ by $A^{*}$ : $W_{0}^{1, q^{\prime}}(\Omega) \rightarrow W^{-1, q^{\prime}}(\Omega)$, and $\mathfrak{a}: W_{0}^{1, q}(\Omega) \times W_{0}^{1, q^{\prime}}(\Omega) \rightarrow \mathbb{R}$ is the bilinear form induced by $A$. Note that $\hat{\mathfrak{a}}: W_{0}^{1, q}(\Omega) \rightarrow \mathbb{R}, \hat{\mathfrak{a}}(v):=\mathfrak{a}(v, v)$ is convex and continuous, and thus weakly lower semi-continuous.

REMARK 2.3 In the case of increased regularity, $a, b, f \in L^{p}(\Omega)$ for $p \geqslant 2$, [15, Thm.II.1] yields that $A y \in L^{p}(\Omega)$ and $\|A y\|_{L^{p}(\Omega)}$ can be bounded by $\|f+u\|_{L^{p}(\Omega)}$ (see [15, Rem.II.2]). In particular, the slack variable $\xi$ satisfies $\xi=A y-u-f \in L^{p}(\Omega)$. Furthermore, if $u+f \in L^{\infty}(\Omega)$, then [48, Rem.2.6] yields Hölder continuity of $y: L^{2}(\Omega) \rightarrow W_{0}^{1, q}(\Omega)$ (or as a mapping to a more regular space, depending on the domain $\Omega$ ) with exponent $\frac{1}{2}$.

The following lemma serves as a tool to prove solvability of the model problem (2.6).

LEMMA 2.4 Let $f \in L^{2}(\Omega)$. If $u_{k} \rightarrow \tilde{u}$ in $L^{2}(\Omega)$, then $\left(y\left(u_{k}\right)\right)_{k \in \mathbb{N}}$ has a subsequence converging weakly in $W_{0}^{1, q}(\Omega)$ to $y(\tilde{u})$.

Proof. The sequence $\left(u_{k}\right)_{k \in \mathbb{N}}$ is bounded owing to its weak convergence. We use the estimate in Proposition 2.1 to obtain the uniform boundedness of $\left(y\left(u_{k}\right)\right)_{k \in \mathbb{N}}$ in $W_{0}^{1, q}(\Omega)$. Next we note that $\left(y_{k}\right)_{k \in \mathbb{N}}$ has a subsequence which converges weakly in $W_{0}^{1, q}(\Omega)$ to a limit $\tilde{y} \in K \cap W_{0}^{1, q}(\Omega)$ due to the weak closedness of $K$. To complete the proof, insert the elements of this subsequence into the variational inequality (2.2) with control $u_{k}$ to show that the limit satisfies $\tilde{y}=y(\tilde{u})$.

Lemma 2.4 and a standard infimizing sequence (Weierstraß) argument now prove the following result.

Proposition 2.5 Problem (2.7) has a solution. 
For $u \in U_{a d}$ of (2.7) we define $y=y(u)$ and $\xi=A y-u-f \in L^{2}(\Omega)$. The set $\{x \in$ $\Omega \mid y(x)=0\}$ is called active set, its complement $\{x \in \Omega \mid y(x)>0\}$ is called inactive set, and the set $\{x \in \Omega \mid \xi(x)=0$ a.e. and $y(x)=0\}$ is called biactive set.

For the derivation of a stationarity system for (2.6) we next study a penalized version of the problem.

\subsection{Penalized variational inequality}

The variational inequality (2.2) can be approximated by the semi-linear partial differential equation

$$
A y-\gamma \max (0,-y)=u+f \quad \text { in } H^{-1}(\Omega),
$$

which is the first order optimality condition of the problem

$$
\text { Minimize } \frac{1}{2}\langle A y, y\rangle_{H^{-1}(\Omega)}-(u+f, y)_{L^{2}(\Omega)}+\frac{\gamma}{2}\|\max (0,-y)\|_{L^{2}(\Omega)}^{2} \text { over } y \in H_{0}^{1}(\Omega) .
$$

Above, $\gamma>0$ is given and $\max (0, \cdot)$ is understood in the pointwise a.e. sense. Equation (2.8) has a unique solution $y_{\gamma}^{\max }(u) \in H_{0}^{1}(\Omega)$. According to [21, Thm. 3.1] it holds for all $u \in L^{2}(\Omega)$ that

$$
y_{\gamma}^{\max }(u) \rightarrow y(u) \quad \text { in } H_{0}^{1}(\Omega) \text { as } \gamma \rightarrow \infty .
$$

In a smoothed version of $(2.8)$, the $\max (0, \cdot)$-operator is approximated by a $C^{1}$-function $\max _{\epsilon}(0, \cdot)$, which depends on a parameter $\epsilon>0$, such that

$$
\max _{\epsilon}(0, \cdot) \rightarrow \max (0, \cdot) \text { pointwise a.e., as } \epsilon \rightarrow 0 .
$$

For now we consider the local variant from [29, Eq. (2.4)], i.e.

$$
\max _{\epsilon}(0, t)=\left\{\begin{array}{cl}
t & \text { if } t \geqslant \epsilon, \\
\frac{t^{2}}{4 \epsilon}+\frac{t}{2}+\frac{\epsilon}{4} & \text { if } t \in(-\epsilon, \epsilon), \\
0 & \text { if } t \leqslant-\epsilon .
\end{array}\right.
$$

The smoothed penalized constraint then reads

$$
A y-\gamma \max _{\epsilon}(0,-y)=u+f \quad \text { in } H^{-1}(\Omega) .
$$

Proposition 2.6 (Solution operator of the semi-linear equation (2.12)) For $\gamma, \epsilon>0, \varphi \in L^{2}(\Omega)$, the linear $H_{0}^{1}(\Omega)$-elliptic partial differential operator $A$ as defined in (2.1) and $\max _{\epsilon}$ from (2.11), the equation

$$
A y-\gamma \max _{\epsilon}(0,-y)=\varphi
$$

admits a unique solution $\tilde{y} \in W_{0}^{1, q}(\Omega)$. Furthermore there exists a constant $C>0$ such that the following estimate holds true:

$$
\max \left\{\gamma\left\|\max _{\epsilon}(0,-\tilde{y})\right\|_{L^{2}(\Omega)},\|\tilde{y}\|_{W_{0}^{1, q}(\Omega)}\right\} \leqslant C\left(\|\varphi\|_{L^{2}(\Omega)}+\gamma \epsilon\right),
$$

from some constant $C=C(\Omega)>0$ which is independent of $\gamma$ and $\epsilon$. 
Proof. To analyze the solvability of the semi-linear equation (2.12), we define $\psi_{\epsilon}: H_{0}^{1}(\Omega) \rightarrow \mathbb{R}$ via

$$
\psi_{\epsilon}(y)=\int_{\Omega} \int_{0}^{y(x)} \max _{\epsilon}(0,-s) d s d x
$$

This mapping is monotone and bounded from below, and its derivative reads

$$
\frac{d}{d y} \psi_{\epsilon}(y) \cdot \phi=-\int_{\Omega} \max _{\epsilon}(0,-y) \phi d x .
$$

Now equation (2.12) can be interpreted as the optimality system of the problem

$$
\text { Minimize } F(y):=\frac{1}{2}\langle A y, y\rangle_{H^{-1}(\Omega)}-\langle\varphi, y\rangle_{H^{-1}(\Omega)}+\gamma \psi_{\epsilon}(y) \text { over } y \in H_{0}^{1}(\Omega),
$$

which has a unique solution $\tilde{y}$, because $F$ is strictly convex and coercive in $H_{0}^{1}(\Omega)$. For all $\epsilon \geqslant 0$, it holds that $\max _{\epsilon}(0,-\tilde{y}) \in H^{1}(\Omega)$. Therefore the function $\tilde{y}_{\tilde{f}}$ can be interpreted as a solution to the linear elliptic equation $A y=\tilde{f}$ with the right hand side $\tilde{f}=\gamma \max _{\epsilon}(0,-\tilde{y})+\varphi \in L^{2}(\Omega)$. According to the proof of Proposition 2.1 this yields that $\tilde{y} \in W_{0}^{1, q}(\Omega)$ and

$$
\|\tilde{y}\|_{W_{0}^{1, q}(\Omega)}=\left\|A^{-1}\left(\gamma \max _{\epsilon}(0,-\tilde{y})+\varphi\right)\right\|_{W_{0}^{1, q}(\Omega)} \leqslant C\left(\left\|\gamma \max _{\epsilon}(0,-\tilde{y})\right\|_{L^{2}(\Omega)}+\|\varphi\|_{L^{2}(\Omega)}\right),
$$

where $A^{-1}$ denotes the solution operator associated with $A v=\tilde{f}, v \in W_{0}^{1, q}(\Omega)$. Next, we establish the bound on $\left\|\gamma \max _{\epsilon}(0,-\tilde{y})\right\|_{L^{2}(\Omega)}$. The semi-linear partial differential equation (2.13) holds in $W^{-1, q}(\Omega)$ and, owing to $\tilde{f}=\gamma \max _{\epsilon}(0,-\tilde{y})+\varphi \in L^{2}(\Omega)$, even in $L^{2}(\Omega)$.

Since $\max (0,-\tilde{y}) \in H_{0}^{1}(\Omega)$ and applying Green's theorem we get from (2.13)

$$
\begin{aligned}
\gamma\|\max (0,-\tilde{y})\|_{L^{2}(\Omega)}^{2} & \leqslant \gamma \int_{\Omega} \max _{\epsilon}(0,-\tilde{y}) \max (0,-\tilde{y}) d x \\
& =-\int_{\Omega} g(x) \nabla \tilde{y}(x)^{T}\left(a_{i j}(x)\right) \nabla \tilde{y}(x) d x-\int_{\Omega} \varphi \max (0,-\tilde{y}) d x,
\end{aligned}
$$

where we also use that $0 \leqslant \max (0,-\tilde{y}) \leqslant \max _{\epsilon}(0,-\tilde{y})$ a.e. in $\Omega$. Here, $0 \leqslant g(x) \in$ $\partial \max (0,-\tilde{y}(x))$ for a.e. $x \in \Omega$, where $\partial$ denotes the subdifferential from convex analysis, cf. [14, Prop.6.45]. The positive definiteness of $\left(a_{i j}\right)$ a.e. in $\Omega$ and Hölder's inequality then yield

$$
\gamma\|\max (0,-\tilde{y})\|_{L^{2}(\Omega)} \leqslant\|\varphi\|_{L^{2}(\Omega)} .
$$

Next we observe that

$$
\begin{aligned}
\left\|\max _{\epsilon}(0,-\tilde{y})\right\|_{L^{2}(\Omega)} & \leqslant\|\max (0,-\tilde{y})\|_{L^{2}(\Omega)}+\left\|\max _{\epsilon}(0,-\tilde{y})-\max (0,-\tilde{y})\right\|_{L^{2}(\Omega)} \\
& \leqslant \gamma^{-1}\|\varphi\|_{L^{2}(\Omega)}+\frac{\epsilon}{4}|\Omega|^{1 / 2} .
\end{aligned}
$$

From (2.15) we consequently obtain

$$
\|\tilde{y}\|_{W_{0}^{1, q}(\Omega)} \leqslant C\left(\|\varphi\|_{L^{2}(\Omega)}+\gamma \epsilon\right)
$$

from some constant $C=C(\Omega)>0$ which is independent of $\gamma$ and $\epsilon$. 
As in [29] we invoke the following assumption on $\gamma$ and $\epsilon$, and we denote the solution operator of (2.12) with $\epsilon=\epsilon(\gamma)$ by $y_{\gamma}: L^{2}(\Omega) \rightarrow H_{0}^{1}(\Omega)$.

Assumption 2.7 For each $\gamma>0$, let $\epsilon(\gamma)>0$ be given such that $\lim _{\gamma \rightarrow \infty} \gamma \epsilon(\gamma)=0$.

THEOREM 2.8 Let $\left(u_{k}\right)_{k \in \mathbb{N}}$ be a sequence in $U_{a d}$ converging weakly in $L^{2}(\Omega)$ to $\tilde{u} \in U_{a d}$. Assume furthermore that $\left(\gamma_{k}\right)_{k \in \mathbb{N}} \subset \mathbb{R}^{>0}$ with $\gamma_{k} \rightarrow \infty$ and let $\epsilon_{k}=\epsilon\left(\gamma_{k}\right)$ satisfy Assumption 2.7. By $y_{k}=y_{\gamma_{k}}\left(u_{k}\right)$ and $\tilde{y}=y(\tilde{u})$ we denote the solution of the smoothed penalized equation (2.12) and the variational inequality (2.2), respectively. For each $k \in \mathbb{N}$ we define $\xi_{k}:=\gamma_{k} \max _{\epsilon_{k}}\left(0,-y_{k}\right)$ and set $\tilde{\xi}:=A \tilde{y}-\tilde{u}-f$. Then there exists a subsequence of $\left(\xi_{k}\right)_{k \in \mathbb{N}}$ and $\left(y_{k}\right)_{k \in \mathbb{N}}$ (denoted the same) such that for $k \rightarrow \infty$,

$$
\xi_{k} \rightarrow \tilde{\xi} \quad \text { in } L^{2}(\Omega), \quad y_{\gamma_{k}}\left(u_{k}\right) \rightarrow y(\tilde{u}) \quad \text { in } W_{0}^{1, q}(\Omega) .
$$

Proof. Theorem 2.3 in [29] provides the strong convergence $y_{k} \rightarrow \tilde{y}$ in $H_{0}^{1}(\Omega)$. The uniform boundedness of the weakly convergent sequence $\left(\left\|u_{k}\right\|_{L^{2}(\Omega)}\right)_{k \in \mathbb{N}}$, together with Proposition 2.6 and the boundedness of the product $\gamma_{k} \epsilon_{k}$, yields a uniform bound on $\left\|\xi_{k}\right\|_{L^{2}(\Omega)}$, and thus a subsequence converging weakly to the limit $\tilde{\xi}=A \tilde{y}-\tilde{u}-f$ in $L^{2}(\Omega)$. Owing to the compact embedding of $L^{2}(\Omega)$ into $W^{-1, q}(\Omega)$, this convergence and also the convergence of $\left(u_{k}\right)_{k \in \mathbb{N}}$ is strong in $W^{-1, q}(\Omega)$. The continuity of $A^{-1}: W^{-1, q}(\Omega) \rightarrow W_{0}^{1, q}(\Omega)$ (see Remark 2.2) then implies strong convergence of the subsequence $\left(y_{k}\right)_{k \in \mathbb{N}}$ in $W_{0}^{1, q}(\Omega)$.

\subsection{Penalized optimal control problem with smoothed objective}

We define the smoothed penalized optimal control problem with smoothed objective as follows:

$$
\begin{gathered}
\text { Minimize } J_{r}(y, u)=\sum_{w \in I} \frac{1}{2\left|B_{r}(w)\right|}\left\|y-y_{w}\right\|_{L^{2}\left(B_{r}(w)\right)}^{2}+\frac{v}{2}\|u\|_{L^{2}(\Omega)}^{2} \\
\quad \text { over }(y, u) \in H_{0}^{1}(\Omega) \times U_{a d} \\
\text { s.t. } A y-\gamma \max _{\epsilon}(0,-y)=u+f \quad \text { in } H^{-1}(\Omega),
\end{gathered}
$$

where $r>0$ is sufficiently small and $B_{r}(w)=\{x \in \Omega|| x-w \mid<r\}$. For each $r>0, J_{r}$ is weakly lower semi-continuous. The smoothing of the objective in the sense of (2.17a) is used below in order to establish a suitable stationarity system for (2.6). In this section, we show that the averaged smooth-penalty scheme (2.17) is consistent with the optimal control problem (2.6) in the sense of Theorem 2.11 below.

Proposition 2.9 For all $\gamma>0, \epsilon>0$ and $r>0$, problem (2.17) has a solution.

Proof. The functional $J_{r}$ is bounded from below. Therefore the set

$$
\left\{J_{r}(y, u) \mid(y, u) \in H_{0}^{1}(\Omega) \times U_{a d} \text { solving }(2.17 \mathrm{c})\right\}
$$

has an infimum denoted by $j$, and we can choose an infimizing sequence $\left(y_{k}, u_{k}\right)_{k \in \mathbb{N}}$ with limit $\lim _{k \rightarrow \infty} J_{r}\left(y_{k}, u_{k}\right)=j$. The sequence $\left(u_{k}\right)_{k \in \mathbb{N}}$ is uniformly bounded in $L^{2}(\Omega)$ and therefore contains a subsequence, which we also denote by $\left(u_{k}\right)$, with weak limit $u \in U_{a d}$. Together with $\left(\left\|u_{k}\right\|_{L^{2}(\Omega)}\right)_{k \in \mathbb{N}}$, by Proposition 2.6, the sequence $\left(\left\|y_{k}\right\|_{W_{0}^{1, q}(\Omega)}\right)_{k \in \mathbb{N}}$ is bounded. By the 
continuous embedding of $W_{0}^{1, q}(\Omega)$ into $H_{0}^{1}(\Omega),\left(\left\|y_{k}\right\|_{H_{0}^{1}(\Omega)}\right)_{k \in \mathbb{N}}$ is also bounded and thus contains a weakly convergent subsequence with limit $y \in H_{0}^{1}(\Omega)$. The limiting pair $(\tilde{y}, \tilde{u})$ is feasible for problem (2.17). The weak lower semi-continuity of the objective $J_{r}$ finally implies

$$
j=\liminf \left\{J_{r}\left(y_{k}, u_{k}\right) \mid k \in \mathbb{N}\right\} \geqslant J_{r}(\tilde{y}, \tilde{u}) .
$$

LEMmA 2.10 Let the sequence $\left(r_{k}\right)_{k \in \mathbb{N}} \subset \mathbb{R}^{>0}$ converge to zero and $\left(G_{k}\right)_{k \in \mathbb{N}} \subset C^{0}(\bar{\Omega})$ converge to $G$ in $C^{0}(\bar{\Omega})$. Then for every $w \in \Omega$,

$$
\frac{1}{\left|B_{r_{k}}(w)\right|} \int_{B_{r_{k}}(w)} G_{k}(x) d x \rightarrow G(w) .
$$

Proof. For every $k \in \mathbb{N}$, we define the mapping

$$
F_{k}: C^{0}(\bar{\Omega}) \rightarrow \mathbb{R}, \quad g \mapsto \frac{1}{\left|B_{r_{k}}(w)\right|} \int_{B_{r_{k}}(w)} g(x) d x,
$$

which is linear and bounded and thus an element of the dual space $C^{0}(\bar{\Omega})^{*}$. Every point $w \in \Omega$ is a Lebesgue point of the continuous function $g \in C^{0}(\bar{\Omega})$, i.e. for $k \rightarrow \infty$

$$
\left\langle F_{k}, g\right\rangle_{C^{0}(\bar{\Omega})^{*}}=\frac{1}{\left|B_{r_{k}}(w)\right|} \int_{B_{r_{k}}(w)} g(x) d x \rightarrow g(w)=\left\langle\delta_{w}, g\right\rangle_{C^{0}(\bar{\Omega})^{*}}
$$

From this we conclude $F_{k} \rightarrow^{*} \delta_{w}$ in $C^{0}(\bar{\Omega})^{*}$. Together with the strong convergence of $G_{k}$ in $C^{0}(\bar{\Omega})$, this yields the convergence of the product

$$
\left\langle F_{k}, G_{k}\right\rangle_{C^{0}(\bar{\Omega})^{*}} \rightarrow\left\langle\delta_{w}, G\right\rangle_{C^{0}(\bar{\Omega})^{*}}=G(w) .
$$

In order to formulate the central theorem on consistency in this section, we denote a solution of the smoothed penalized problem (2.17) with parameters $(\gamma, \epsilon, r)=\left(\gamma_{k}, \epsilon_{k}, r_{k}\right)$ by $\left(y_{k}, u_{k}\right)$.

THEOREM 2.11 Assume that $\left(\gamma_{k}\right)_{k \in \mathbb{N}},\left(r_{k}\right)_{k \in \mathbb{N}} \subset \mathbb{R}^{>0}$ converge to zero and let $\epsilon_{k}=\epsilon\left(\gamma_{k}\right)$ satisfy Assumption 2.7. Then there exists a subsequence of $\left(y_{k}, u_{k}\right)_{k \in \mathbb{N}}$ (denoted the same) and a solution $(\tilde{y}, \tilde{u})$ of the original problem (2.7) such that

$$
y_{k} \rightarrow \tilde{y} \quad \text { in } W_{0}^{1, q}(\Omega), \quad u_{k} \rightarrow \tilde{u} \quad \text { in } L^{2}(\Omega) .
$$

Proof. We choose $\bar{u} \in U_{a d}$ and denote the solution of the semi-linear equation (2.17c) with $\gamma=\gamma_{k}$ and $\epsilon=\epsilon_{k}$ by $y_{\gamma_{k}}(\bar{u})$ for all $k \in \mathbb{N}$. Proposition 2.6, together with Assumption 2.7, yields a bound on $\left(\left\|y_{\gamma_{k}}(\bar{u})\right\|_{W_{0}^{1, q}(\Omega)}\right)_{k \in \mathbb{N}}$ that does not depend on $k \in \mathbb{N}$, and we can estimate

$$
\frac{\alpha}{2}\left\|u_{k}\right\|_{L^{2}(\Omega)}^{2} \leqslant J_{r_{k}}\left(y_{k}, u_{k}\right) \leqslant J_{r_{k}}\left(y_{\gamma_{k}}(\bar{u}), \bar{u}\right) \leqslant C .
$$

The uniform boundedness of $\left(u_{k}\right)_{k \in \mathbb{N}}$ in $L^{2}(\Omega)$ thus yields a subsequence, still denoted by $\left(u_{k}\right)_{k \in \mathbb{N}}$, with weak limit $\tilde{u} \in U_{a d}$. Theorem 2.8 then yields the strong convergence of a (sub-)subsequence $\left(y_{k}\right)_{k \in \mathbb{N}}$ to the solution $\tilde{y}=y(\tilde{u})$ of $(2.2)$ in $W_{0}^{1, q}(\Omega)$. To prove optimality of the limiting pair $(\tilde{y}, \tilde{u})$, let $\left(y^{\star}, u^{\star}\right)$ denote a solution of problem (2.7). Using Lemma 2.10 for the first term in 
$J_{r_{k}}\left(y_{\gamma_{k}}\left(u^{\star}\right), u^{\star}\right)$, as $y_{\gamma_{k}}\left(u^{\star}\right) \rightarrow y\left(u^{\star}\right)$ in $C^{0}(\bar{\Omega})$ by Theorem 2.8 and the embedding of $W_{0}^{1, q}(\Omega)$ into $C^{0}(\bar{\Omega})$, we observe that

$$
\liminf _{k \rightarrow \infty} J_{r_{k}}\left(y_{\gamma_{k}}\left(u^{\star}\right), u^{\star}\right)=\lim _{k \rightarrow \infty} J_{r_{k}}\left(y_{\gamma_{k}}\left(u^{\star}\right), u^{\star}\right)=J\left(y\left(u^{\star}\right), u^{\star}\right)=J\left(y^{\star}, u^{\star}\right) .
$$

Furthermore, with the weak lower semi-continuity of the second term in $J_{r_{k}}\left(y_{k}, u_{k}\right)$, and the same arguments for the first term, we note that

$$
\liminf _{k \rightarrow \infty} J_{r_{k}}\left(y_{k}, u_{k}\right) \geqslant J(\tilde{y}, \tilde{u}) .
$$

Now we exploit the optimality of $\left(y_{k}, u_{k}\right)$ with respect to the objective $J_{r_{k}}$ as well as the feasibility of $\left(y_{\gamma_{k}}\left(u^{\star}\right), u^{\star}\right)$ for the smoothed penalized problem to derive $J_{r_{k}}\left(y_{k}, u_{k}\right) \leqslant J_{r_{k}}\left(y_{\gamma_{k}}\left(u^{\star}\right), u^{\star}\right)$ for all $k \in \mathbb{N}$. Altogether it holds that

$$
J(\tilde{y}, \tilde{u}) \leqslant \liminf _{k \rightarrow \infty} J_{r_{k}}\left(y_{k}, u_{k}\right) \leqslant \liminf _{k \rightarrow \infty} J_{r_{k}}\left(y_{\gamma_{k}}\left(u^{\star}\right), u^{\star}\right)=J\left(y^{\star}, u^{\star}\right) .
$$

\section{Derivation of stationarity conditions}

\subsection{Optimality system for the smoothed penalized problem}

For the derivation of a first order optimality system for (2.17) we apply [51, Thm. 3.1]. For this purpose we have to check the associated constraint qualification, i.e. regularity of a solution.

LEMMA 3.1 Every feasible point $\left(y_{k}, u_{k}\right) \in H_{0}^{1}(\Omega) \times L^{2}(\Omega)$ of (2.17) with parameters $\gamma_{k}, \epsilon_{k} \in$ $\mathbb{R}^{>0}$ is regular in the sense of [51].

Proof. For $(y, u) \in \mathcal{X}:=H_{0}^{1}(\Omega) \times L^{2}(\Omega)$, define $g(y, u)=A y-\gamma_{k} \max _{\epsilon_{k}}(0,-y)-u-f$ with Fréchet derivative

$$
g^{\prime}\left(y_{k}, u_{k}\right)(y, u)=A y+\gamma_{k} \max _{\epsilon_{k}}^{\prime}\left(0,-y_{k}\right) y-u .
$$

We formulate (2.17c) as $g(y, u) \in\{0\} \subset H^{-1}(\Omega)$ and, according to [51, Equ. (1.4)], have to show that

$$
g^{\prime}\left(y_{k}, u_{k}\right) \mathcal{C}\left(y_{k}, u_{k}\right)=H^{-1}(\Omega),
$$

where $\mathrm{C}\left(y_{k}, u_{k}\right):=\left\{t\left((y, u)-\left(y_{k}, u_{k}\right)\right) \mid t \geqslant 0,(y, u) \in H_{0}^{1}(\Omega) \times U_{a d}\right\}$. Note that the definitions of $A$ and $\max _{\epsilon_{k}}$ in (2.1) and (2.11) yield immediately that $g^{\prime}\left(y_{k}, u_{k}\right) \mathrm{e}\left(y_{k}, u_{k}\right) \subset H^{-1}(\Omega)$. Furthermore, the operator $g^{\prime}\left(y_{k}, u_{k}\right): \mathcal{C}\left(y_{k}, u_{k}\right) \rightarrow H^{-1}(\Omega)$ is surjective, because for any given $\phi \in H^{-1}(\Omega)$, we can choose $u=u_{k} \in U_{a d}$ and $y=\tilde{y}+y_{k} \in H_{0}^{1}(\Omega)$, where $\tilde{y}$ is the solution of the linear elliptic partial differential equation $A y+\gamma_{k} \max _{\epsilon_{k}}^{\prime}\left(0,-y_{k}\right) y=\phi$. Then with $t=1$, $(\tilde{y}, 0) \in \mathrm{C}\left(y_{k}, u_{k}\right)$ and $g^{\prime}\left(y_{k}, u_{k}\right)(\tilde{y}, 0)=\phi$.

As a consequence of [51, Thm. 3.1] and Lemma 3.1 we obtain stationarity conditions for the smoothed penalized problem (2.17) as stated in the next proposition.

PROPOSITION 3.2 If $\left(y_{k}, u_{k}\right) \in H_{0}^{1}(\Omega) \times U_{a d}$ with $U_{a d} \neq L^{2}(\Omega)$ is optimal for problem (2.17) with parameters $\left(\gamma_{k}, \epsilon_{k}, r_{k}\right) \in\left(\mathbb{R}^{>0}\right)^{3}$, then there exists $p_{k} \in H_{0}^{1}(\Omega)$ such that the following first 
order conditions hold:

$$
\begin{aligned}
u_{k}=\frac{1}{v} p_{k}+\left(a-\frac{1}{v} p_{k}\right)_{+}-\left(\frac{1}{v} p_{k}-b\right)_{+} & \text {in } L^{2}(\Omega), \\
A^{*} p_{k}+\gamma_{k} \max _{\epsilon_{k}}^{\prime}\left(0,-y_{k}\right) p_{k}=-\sum_{w \in I} \frac{1}{\left|B_{r_{k}}(w)\right|}\left(y_{k}-y_{w}\right) \chi_{B_{r_{k}}(w)} & \text { in } H^{-1}(\Omega) .
\end{aligned}
$$

If $U_{a d}=L^{2}(\Omega)$, then (3.2a) is replaced by $u_{k}=\frac{1}{v} p_{k}$.

REMARK 3.3 We introduce the variable $\sigma_{k}=v u_{k}-p_{k} \in L^{2}(\Omega)$, which can be decomposed as $\sigma_{k}=\sigma_{a k}-\sigma_{b k}$ with $\sigma_{a k}=\max \left(0, \sigma_{k}\right)$ and $\sigma_{b k}=-\min \left(0, \sigma_{k}\right)$ pointwise almost everywhere. Then the following conditions are equivalent to (3.2a):

$$
\begin{aligned}
\left(v u_{k}, u-u_{k}\right)_{L^{2}(\Omega)}-\left(p_{k}, u-u_{k}\right)_{L^{2}(\Omega)} & \geqslant 0 \quad \forall u \in U_{a d}, \\
u_{k} & =\Pi_{U_{a d}}\left(\frac{1}{v} p_{k}\right), \\
u_{k} \in U_{a d}, \quad \sigma_{a k} \geqslant 0, \quad \sigma_{b k} \geqslant 0, \quad \sigma_{a k}\left(a-u_{k}\right) & =\sigma_{b k}\left(b-u_{k}\right)=0 .
\end{aligned}
$$

Here $\Pi_{U_{a d}}$ denotes the $L^{2}(\Omega)$-projection into the closed convex set $U_{a d}$.

\subsection{Stationarity system for the optimal control problem}

Borrowing terminology from [28], next we define the stationarity concept which is relevant in our context.

DEFinition 3.4 (Limiting $\varepsilon$-almost C-stationarity) We call $(y, u, \xi) \in W_{0}^{1, q}(\Omega) \times U_{a d} \times$ $W^{-1, q}(\Omega), U_{a d} \neq L^{2}(\Omega)$ limiting $\varepsilon$-almost C-stationary for problem (2.6) if $y=y(u)$ solves the variational inequality (2.2), $\xi$ is defined as $\xi=A y-u-f$, and if there exist $p \in W_{0}^{1, q^{\prime}}(\Omega)$, $\lambda \in m_{b}(\bar{\Omega})$ and sequences $\left(p_{k}\right)_{k \in \mathbb{N}} \subset H_{0}^{1}(\Omega)$ with $p_{k} \rightarrow p$ in $W_{0}^{1, q^{\prime}}(\Omega)$, and $\left(\lambda_{k}\right)_{k \in \mathbb{N}} \subset$ $H^{-1}(\Omega)$ with $\lambda_{k} \rightarrow^{*} \lambda$ in $m_{b}(\bar{\Omega})$ such that the following conditions are satisfied,

$$
\begin{aligned}
u-\frac{1}{v} p-\left(a-\frac{1}{v} p\right)_{+}+\left(\frac{1}{v} p-b\right)_{+} & =0 \\
\forall \psi \in W_{0}^{1, q}(\Omega):\left\langle\psi, A^{*} p\right\rangle_{W_{0}^{1, q}(\Omega)}-\langle\psi, \lambda\rangle_{W_{0}^{1, q}(\Omega)}+\sum_{w \in I}\left(y(w)-y_{w}\right) \psi(w) & =0, \\
\langle\lambda, y\rangle_{W^{-1, q^{\prime}}(\Omega)} & =0, \\
\forall \tau>0 \quad \exists E_{\tau} \subset\{y>0\} \text { such that }\left|\{y>0\} \backslash E_{\tau}\right|<\tau \text { and } & \text { (3.6b) } \\
\forall \varphi \in C^{0}(\bar{\Omega}),\left.\varphi\right|_{\Omega \backslash E_{\tau}}=0, \quad\langle\lambda, \varphi\rangle_{m_{b}(\bar{\Omega})} & =0, \\
\lim \sup \left\{\left\langle\lambda_{k}, p_{k}\right\rangle_{H^{-1}(\Omega)} \mid k \in \mathbb{N}\right\} & \leqslant 0, \\
\langle\xi, p\rangle_{W^{-1, q}(\Omega)} & =0 .
\end{aligned}
$$

If $U_{a d}=L^{2}(\Omega)$, then (3.6a) is again replaced by $u=\frac{1}{v} p \in W_{0}^{1, q^{\prime}}(\Omega)$. 
THEOREM 3.5 For each $k \in \mathbb{N}$, let $\gamma_{k}, \epsilon_{k}=\epsilon\left(\gamma_{k}\right), r_{k}>0$ be penalization and smoothing parameters which satisfy Assumption 2.7, where $\gamma_{k} \rightarrow \infty$ and $r_{k} \rightarrow 0$.

Furthermore let $\left(y_{k}, u_{k}, p_{k}\right)$ be stationary for problem (2.17) in the sense that the tuple is feasible and (3.2a), (3.2b) hold, and assume that $\left(\left\|u_{k}\right\|_{L^{2}(\Omega)}\right)_{k \in \mathbb{N}}$ is bounded.

Then there exist $(\tilde{y}, \tilde{u}, \tilde{\xi}, \tilde{p}, \tilde{\lambda}) \in W_{0}^{1, q}(\Omega) \times L^{2}(\Omega) \times L^{2}(\Omega) \times H_{0}^{1}(\Omega) \times m_{b}(\bar{\Omega})$ and a subsequence (also denoted by index $k$ ) such that

$$
\begin{aligned}
y_{k} & \rightarrow \tilde{y} & & \text { in } W_{0}^{1, q}(\Omega), \\
u_{k} & \rightarrow \tilde{u} & & \text { in } L^{2}(\Omega), \\
\xi_{k}:=\gamma_{k} \max _{\epsilon_{k}}\left(0,-y_{k}\right) & -\tilde{\xi} & & \text { in } L^{2}(\Omega), \\
p_{k} & -\tilde{p} & & \text { in } H_{0}^{1}(\Omega), \\
\lambda_{k}:=-\gamma_{k} \max _{\epsilon_{k}}^{\prime}\left(0,-y_{k}\right) p_{k} & { }^{*} \tilde{\lambda} & & \text { in } C^{0}(\bar{\Omega})^{*},
\end{aligned}
$$

and $(\tilde{y}, \tilde{u}, \tilde{\xi})$ is a limiting $\varepsilon$-almost C-stationary point for problem (2.6) with multipliers $\tilde{p}, \tilde{\lambda}$.

Proof. Convergence and feasibility of $y_{k}, u_{k}, \xi_{k}$ and $p_{k}$. Theorem 2.8 yields the assertions for $y_{k}$ and $\xi_{k}$. Testing the adjoint equation with $p_{k} \in H_{0}^{1}(\Omega)$, we obtain a uniform bound on $\left(\left\|p_{k}\right\|_{H_{0}^{1}(\Omega)}\right)_{k \in \mathbb{N}}$ as follows,

$$
\begin{aligned}
C\left\|p_{k}\right\|_{H_{0}^{1}(\Omega)}^{2} & \leqslant\left\langle p_{k}, A^{*} p_{k}\right\rangle_{H^{-1}(\Omega)} \\
& =-\gamma_{k} \int_{\Omega} \max _{\epsilon_{k}}^{\prime}\left(0,-y_{k}\right) p_{k}^{2} d x-\sum_{w \in I} \frac{1}{\left|B_{r_{k}}(w)\right|} \int_{B_{r_{k}}(w)}\left(y_{k}-y_{w}\right) p_{k} d x \\
& \leqslant C\left(\operatorname{card}(I)\left\|y_{k}\right\|_{L^{\infty}(\Omega)}+\sup \left\{\left|y_{w}\right| \mid w \in I\right\}\right)\left\|p_{k}\right\|_{L^{2}(\Omega)} .
\end{aligned}
$$

The weak convergence of a subsequence of $\left(u_{k}\right)_{k \in \mathbb{N}}$ in $L^{2}(\Omega)$ follows from the boundedness of the operator $(\cdot)_{+}: L^{2}(\Omega) \rightarrow L^{2}(\Omega)$. Note that if $a, b \in H^{1}(\Omega)$, and $\left.a\right|_{\partial \Omega}<0<\left.b\right|_{\partial \Omega}$, then we have convergence along a subsequence in $H_{0}^{1}(\Omega)$.

Convergence of $\lambda_{k}$. For $\delta>0$ we define the function

$$
\rho_{\delta}: \mathbb{R} \rightarrow \mathbb{R}, \quad \rho_{\delta}(p)=\left\{\begin{array}{cl}
-1 & \text { for } p<-\delta, \\
\frac{p}{\delta} & \text { for } p \in[-\delta, \delta], \\
1 & \text { for } p>\delta,
\end{array}\right.
$$

and note that $\lim _{\delta \rightarrow 0+} \rho_{\delta}(p)=\operatorname{sign}(p)$ for all $p \in \mathbb{R}$. Furthermore, $\rho_{\delta}\left(p_{k}\right) \in H_{0}^{1}(\Omega) \cap L^{\infty}(\Omega)$ is a feasible test function for the adjoint equation (3.2b), yielding

$$
\begin{aligned}
& \left\langle A^{*} p_{k}, \rho_{\delta}\left(p_{k}\right)\right\rangle_{H^{-1}(\Omega)}+\gamma_{k} \int_{\Omega} \max _{\epsilon_{k}}^{\prime}\left(0,-y_{k}\right) p_{k} \rho_{\delta}\left(p_{k}\right) d x \\
& =-\sum_{w \in I} \frac{1}{\left|B_{r_{k}}(w)\right|} \int_{B_{r_{k}}(w)}\left(y_{k}-y_{w}\right) \rho_{\delta}\left(p_{k}\right) d x \leqslant \operatorname{card}(I)\left\|y_{k}\right\|_{L^{\infty}(\Omega)}+\sup \left\{\left|y_{w}\right| \mid w \in I\right\} \leqslant C .
\end{aligned}
$$

The first term on the left hand side is non-negative as

$$
\left\langle A^{*} p_{k}, \rho_{\delta}\left(p_{k}\right)\right\rangle_{H^{-1}(\Omega)} \geqslant C \int_{\Omega} \rho_{\delta}^{\prime}\left(p_{k}\right)\left|\nabla p_{k}\right|^{2} d x \geqslant 0 .
$$


This yields the uniform boundedness of $\gamma_{k} \int_{\Omega} \max _{\epsilon_{k}}^{\prime}\left(0,-y_{k}\right) p_{k} \rho_{\delta}\left(p_{k}\right) d x \geqslant 0$. Sending $\delta \rightarrow 0$, we obtain the bound

$$
\left\|\lambda_{k}\right\|_{L^{1}(\Omega)}=\int_{\Omega}\left|\lambda_{k}\right| d x=\lim _{\delta \rightarrow 0} \int_{\Omega} \gamma_{k} \max _{\epsilon_{k}}^{\prime}\left(0,-y_{k}\right) p_{k} \rho_{\delta}\left(p_{k}\right) d x \leqslant C .
$$

The embedding of $L^{1}(\Omega)$ into $m_{b}(\bar{\Omega})=C^{0}(\bar{\Omega})^{*}$ and the Banach-Alaoglu theorem then provides the existence of a subsequence converging weakly* to $\tilde{\lambda} \in m_{b}(\bar{\Omega})$. Note that because of the embedding of $W_{0}^{1, q}(\Omega)$ into $C^{0}(\bar{\Omega})$, the subsequence also converges weakly in $W^{-1, q^{\prime}}(\Omega)$ and $\left(\lambda_{k}\right)_{k \in \mathbb{N}}$ is bounded in this space.

Adjoint equation (3.6b) for $\tilde{y}, \tilde{p}, \tilde{\lambda}$. Since $p_{k} \rightarrow \tilde{p}$ in $W_{0}^{1, q^{\prime}}(\Omega) \supset H_{0}^{1}(\Omega)$, so by the properties of $A$ we have $A^{*} p_{k} \rightarrow A^{*} \tilde{p}$ in $W^{-1, q^{\prime}}(\Omega)$. If $\varphi \in C^{0}(\bar{\Omega})$, then $\sum_{w \in I}\left(y_{k}-y_{w}\right) \varphi \rightarrow \sum_{w \in I}(\tilde{y}-$ $\left.y_{w}\right) \varphi$ in $C^{0}(\bar{\Omega})$ and by Lemma 2.10 ,

$$
\begin{aligned}
\left\langle\sum_{w \in I} \frac{1}{\left|B_{r_{k}}(w)\right|}\left(y_{k}-y_{w}\right) \chi_{B_{r_{k}}(w)}, \varphi\right\rangle_{C^{0}(\bar{\Omega})^{*}} & =\sum_{w \in I} \frac{1}{\left|B_{r_{k}}(w)\right|} \int_{B_{r_{k}}(w)}\left(y_{k}-y_{w}\right) \varphi d x \\
& \rightarrow \sum_{w \in I}\left(\tilde{y}(w)-y_{w}\right) \varphi(w) .
\end{aligned}
$$

The embedding of $C^{0}(\bar{\Omega})^{*}$ into $W^{-1, q^{\prime}}(\Omega)$ then gives

$$
\sum_{w \in I} \frac{1}{\left|B_{r_{k}}(w)\right|}\left(y_{k}-y_{w}\right) \chi_{B_{r_{k}}(w)}-\sum_{w \in I}\left(\tilde{y}-y_{w}\right) \delta_{w} \quad \text { in } W^{-1, q^{\prime}}(\Omega) .
$$

Together with the weak convergence of $\lambda_{k}$ in $W^{-1, q^{\prime}}(\Omega)$, this yields equation (3.6b).

Complementarity of $\tilde{y}$ and $\tilde{\lambda}$ (3.6c). From the convergence $y_{k} \rightarrow \tilde{y}$ in $W_{0}^{1, q}(\Omega)$ we infer that $\left(-y_{k}\right)_{+} \rightarrow(-\tilde{y})_{+}=0$ in $W_{0}^{1, q}(\Omega)$, so

$$
\left\langle\lambda_{k},\left(-y_{k}\right)_{+}\right\rangle_{W^{-1, q^{\prime}}(\Omega)} \rightarrow\langle\tilde{\lambda}, 0\rangle_{W^{-1, q^{\prime}}(\Omega)}=0 .
$$

Furthermore we observe that

$$
\begin{aligned}
\left|\left\langle\lambda_{k},\left(y_{k}\right)_{+}\right\rangle_{W^{-1, q^{\prime}(\Omega)}}\right| & =\gamma_{k} \int_{\Omega} \max _{\epsilon_{k}}^{\prime}\left(0,-y_{k}\right)\left|p_{k}\right|\left(y_{k}\right)_{+} d x \\
& =\gamma_{k} \int_{\left\{0 \leqslant y_{k} \leqslant \epsilon_{k}\right\}} \max _{\epsilon_{k}}^{\prime}\left(0,-y_{k}\right)\left|p_{k}\right| y_{k} d x+\gamma_{k} \int_{\left\{y_{k}>\epsilon_{k}\right\}} 0 d x \\
& \leqslant \gamma_{k} \epsilon_{k}\left\|p_{k}\right\|_{L^{1}(\Omega)},
\end{aligned}
$$

which converges to zero due to the boundedness of $\left(p_{k}\right)_{k \in \mathbb{N}}$ in $W_{0}^{1, q^{\prime}}(\Omega)$ and Assumption 2.7. So decomposing $y_{k}=\left(y_{k}\right)_{+}-\left(-y_{k}\right)_{+}$we get

$$
\left\langle\lambda_{k}, y_{k}\right\rangle_{W^{-1, q^{\prime}}(\Omega)}=\left\langle\lambda_{k},\left(y_{k}\right)_{+}\right\rangle_{W^{-1, q^{\prime}}(\Omega)}-\left\langle\lambda_{k},\left(-y_{k}\right)_{+}\right\rangle_{W^{-1, q^{\prime}}(\Omega)} \rightarrow 0 .
$$

We also have the convergence $\left\langle\lambda_{k}, y_{k}\right\rangle_{W^{-1, q^{\prime}}(\Omega)} \rightarrow\langle\tilde{\lambda}, \tilde{y}\rangle_{W^{-1, q^{\prime}}(\Omega)}$, which yields the assertion (3.6c). 
Condition (3.6d) Let $\left(\delta_{\ell}\right)_{\ell \in \mathbb{N}} \subset \mathbb{R}^{>0}$ converge monotonically to zero and define $M_{\ell}=\{0<\tilde{y}<$ $\left.\delta_{\ell}\right\}$. For $\ell_{1} \geqslant \ell_{2}, M_{\ell_{1}} \subset M_{\ell_{2}}$ and the intersection $M=\bigcap_{\ell \in \mathbb{N}}\left\{0<\tilde{y}<\delta_{\ell}\right\}$ is empty. Indeed, assume $x \in M$. Then $\forall \ell \in \mathbb{N}, \tilde{y}(x)<\delta_{\ell}$ and thus $\lim _{\ell \rightarrow \infty} \tilde{y}(x) \leqslant \lim _{\ell \rightarrow \infty} \delta_{\ell}=0$. The feasibility of $\tilde{y}$ for (2.2) yields $\tilde{y}(x) \geqslant 0$. But $\tilde{y}(x)=0$ is a contradiction to $x \in M$. This implies that $\left|M_{\ell}\right| \rightarrow 0$, and thus for an arbitrary $\tau>0$ there exists an index $\ell(\tau)$ such that $\left|M_{\ell(\tau)}\right| \leqslant \tau$. Hence $E_{\tau}=\{\tilde{y}>0\} \backslash M_{\ell(\tau)}=\left\{\tilde{y} \geqslant \delta_{\ell(\tau)}\right\}$ fulfills $\left|\{\tilde{y}>0\} \backslash E_{\tau}\right| \leqslant \tau$, and $\Omega \backslash E_{\tau}=\left\{\tilde{y}<\delta_{\ell(\tau)}\right\}$.

Let $\varphi \in C^{0}(\bar{\Omega}),\left.\varphi\right|_{\left\{\tilde{y}<\delta_{\ell(\tau)}\right\}}=0$. The weak* convergence of $\lambda_{k}$ in $m_{b}(\bar{\Omega})$ guarantees

$$
\left\langle\lambda_{k}, \varphi\right\rangle_{m_{b}(\bar{\Omega})} \rightarrow\langle\tilde{\lambda}, \varphi\rangle_{m_{b}(\bar{\Omega})}
$$

For all $k \in \mathbb{N}, \lambda_{k} \in L^{2}(\Omega)$, so we can write the product on the left hand side as the integral

$$
\left\langle\lambda_{k}, \varphi\right\rangle_{m_{b}(\bar{\Omega})}=\int_{\left\{\tilde{y} \geqslant \delta_{\ell(\tau)}\right\}} \lambda_{k} \varphi d x .
$$

We use the strong convergence of $y_{k}$ to $\tilde{y}$ in $C^{0}\left(\left\{\tilde{y} \geqslant \delta_{\ell(\tau)}\right\}\right)$, which comes from the compact embedding of $W_{0}^{1, q}(\Omega)$ into $C^{0}(\bar{\Omega})$, to obtain an index $c_{1}\left(\delta_{\ell(\tau)}\right) \in \mathbb{N}$ such that

$$
\left\|\tilde{y}-y_{k}\right\|_{C^{0}\left(\left\{\tilde{y} \geqslant \delta_{\ell(\tau)}\right\}\right)}<\frac{\delta_{\ell(\tau)}}{2} \quad \forall k \geqslant c_{1}\left(\delta_{\ell(\tau)}\right) .
$$

With a pointwise interpretation this yields for all $x \in\left\{\tilde{y} \geqslant \delta_{\ell(\tau)}\right\}$ and $k \geqslant c_{1}\left(\delta_{\ell(\tau)}\right)$ that $\mid \tilde{y}(x)-$ $y_{k}(x) \mid<\frac{\delta_{\ell(\tau)}}{2}$, and so

$$
y_{k}(x)=\left|y_{k}(x)\right| \geqslant|\tilde{y}(x)|-\left|\tilde{y}(x)-y_{k}(x)\right|>\delta_{\ell(\tau)}-\frac{\delta_{\ell(\tau)}}{2}=\frac{\delta_{\ell(\tau)}}{2} .
$$

Furthermore we use the convergence $\epsilon_{k} \rightarrow 0$ and obtain another index $c_{2}\left(\delta_{\ell(\tau)}\right) \in \mathbb{N}$ such that

$$
\forall k \geqslant c_{2}\left(\delta_{\ell(\tau)}\right), \quad \epsilon_{k}<\frac{\delta_{\ell(\tau)}}{2} .
$$

Then, for all $x \in\left\{\tilde{y} \geqslant \delta_{\ell(\tau)}\right\}$ and $k \geqslant \max \left\{c_{1}\left(\delta_{\ell(\tau)}\right), c_{2}\left(\delta_{\ell(\tau)}\right)\right\}$, we have

$$
-y_{k}(x)<-\frac{\delta_{\ell(\tau)}}{2}<-\epsilon_{k}
$$

and therefore $\max _{\epsilon_{k}}^{\prime}\left(0,-y_{k}(x)\right)=0$. It follows that for $k \geqslant \max \left\{c_{1}\left(\delta_{\ell(\tau)}\right), c_{2}\left(\delta_{\ell(\tau)}\right)\right\}$, the integrand

$$
\lambda_{k} \varphi=-\gamma_{k} \max _{\epsilon_{k}}^{\prime}\left(0,-y_{k}\right) p_{k} \varphi
$$

in (3.9) is zero, and so the integral vanishes.

Sign condition (3.6e) for $\lim \sup \left\{\left\langle\lambda_{k}, p_{k}\right\rangle_{H^{-1}(\Omega)} \mid k \in \mathbb{N}\right\} \quad$ For all $k \in \mathbb{N}$,

$$
\left\langle\lambda_{k}, p_{k}\right\rangle_{H^{-1}(\Omega)}=\left(-\gamma_{k} \max _{\epsilon_{k}}^{\prime}\left(0,-y_{k}\right) p_{k}, p_{k}\right)_{L^{2}(\Omega)}=\int_{\Omega}-\gamma_{k} \max _{\epsilon_{k}}^{\prime}\left(0,-y_{k}\right) p_{k}^{2} d x \leqslant 0 .
$$

This yields the assertion. 
Complementarity $(\tilde{p}, \tilde{\xi})_{L^{2}(\Omega)}=0$ (3.6f) The regularity of $\tilde{\xi} \in L^{2}(\Omega)$ allows us to consider the pairing $\langle\tilde{\xi}, \tilde{p}\rangle_{W^{-1, q}(\Omega)}$ as an integral, whereas the fact that $\left.\tilde{\xi}\right|_{\{\tilde{y} \neq 0\}}=0$ yields $\langle\tilde{\xi}, \tilde{p}\rangle_{W^{-1, q}(\Omega)}=$ $\int_{\Omega} \tilde{\xi} \tilde{p} \chi\{\tilde{y}=0\} d x$. A subsequence of the product $\xi_{k} \chi\{\tilde{y}=0\}$ converges weakly in $L^{2}(\Omega)$ and thus strongly in $W^{-1, q}(\Omega)$ to $\tilde{\xi} \chi\{\tilde{y}=0\}$. Consequently we have

$$
(\tilde{p}, \tilde{\xi})_{L^{2}(\Omega)}=\int_{\{\tilde{y}=0\}} \tilde{p} \tilde{\xi} d x=\lim _{k \rightarrow \infty} \int_{\{\tilde{y}=0\}} \xi_{k} p_{k} d x
$$

along a suitable subsequence. Next we split the integral on the right hand side as follows:

$$
\begin{aligned}
\int_{\{\tilde{y}=0\}} \xi_{k} p_{k} d x=\int_{\{\tilde{y}=0\}} \xi_{k} p_{k} \chi_{\left\{\left|y_{k}\right| \leqslant \epsilon_{k}\right\}} d x+\int_{\{\tilde{y}=0\}} \xi_{k} p_{k} \chi_{\left\{y_{k}>\epsilon_{k}\right\}} d x & \\
& +\int_{\{\tilde{y}=0\}} \xi_{k} p_{k} \chi_{\left\{y_{k}<-\epsilon_{k}\right\}} d x .
\end{aligned}
$$

In the same way as in [29], the first integral converges to zero because

$$
\left\|\xi_{k} \chi_{\left\{\left|y_{k}\right| \leqslant \epsilon_{k}\right\}}\right\|_{L^{2}(\Omega)}^{2}=\int_{\left\{\left|y_{k}\right| \leqslant \epsilon_{k}\right\}} \gamma_{k}^{2}\left(\frac{y_{k}^{2}}{4 \epsilon_{k}}+\frac{y_{k}}{2}+\frac{\epsilon_{k}}{4}\right)^{2} d x \leqslant|\Omega|\left(\gamma_{k} \epsilon_{k}\right)^{2} \rightarrow 0
$$

and $\left\|p_{k}\right\|_{L^{2}(\Omega)}$ is uniformly bounded. The second integral in (3.11) is equal to zero for every $k \in$ $\mathbb{N}$ owing to the definition $\xi_{k}=\gamma_{k} \max _{\epsilon_{k}}\left(0,-y_{k}\right)$ and the fact that $\max _{\epsilon_{k}}\left(0,-y_{k}\right)$ is zero when $-y_{k} \leqslant-\epsilon_{k}$. For the third integral in (3.11) we use the definition of $\xi_{k}$ and $\lambda_{k}$ to obtain

$$
\xi_{k} p_{k} \chi_{\left\{y_{k}<-\epsilon_{k}\right\}}=\gamma_{k} \max _{\epsilon_{k}}\left(0,-y_{k}\right) p_{k} \chi_{\left\{y_{k}<-\epsilon_{k}\right\}}=-\lambda_{k} y_{k} \chi_{\left\{y_{k}<-\epsilon_{k}\right\}}
$$

and estimate

$$
\left|\int_{\{\tilde{y}=0\}} \xi_{k} p_{k} \chi_{\left\{y_{k}<-\epsilon_{k}\right\}} d x\right| \leqslant\left\|\lambda_{k}\right\|_{L^{1}(\Omega)}\left\|y_{k} \chi_{\{\tilde{y}=0\}}\right\|_{L^{\infty}(\Omega)} .
$$

The sequence $\left(\lambda_{k}\right)_{k \in \mathbb{N}}$ is bounded in $L^{1}(\Omega)$ owing to (3.8), and $y_{k} \chi_{\{\tilde{y}=0\}}$ converges strongly in $L^{\infty}(\Omega)$ to $\tilde{y} \chi_{\{\tilde{y}=0\}}=0$. So the third integral in (3.11) also vanishes for $k \rightarrow \infty$, and the representation of $(\tilde{p}, \tilde{\xi})_{L^{2}(\Omega)}$ in (3.10) yields the assertion.

This concludes our analysis of (2.6). In the next section we study numerical aspects of solving (2.6).

\section{Primal-dual weighted error indicator}

This section is devoted to the derivation of an a posteriori error indicator for (limiting $\epsilon$-almost) C-stationary points for problem (2.6) with control constraints. We enhance the concept in [10] and develop a localized a posteriori approximation for the error of a discrete approximate C-stationary point $\left(y_{h}, u_{h}\right)$ compared to the exact solution $(y, u)$ in the objective, i.e., we estimate the value

$$
\left|J\left(y_{h}, u_{h}\right)-J(y, u)\right| .
$$

The structure of the resulting indicator is similar to the one in [27] and it contains primal and dual equations as well as complementarity mismatch terms for the state $y$ and the slack variable $\xi$. 
However, additional difficulties arise from the poor regularity of the dual equation, the occurrence of tracking points and the control constraints. We start by introducing an abstract discretization in Section 4.1, and derive a representation formula for the error in the objective in Section 4.2, cf. [4]. This formula is still flexible with respect to the choice of the finite element scheme. Finally, in Section 4.3 , we apply our formula to a $P^{1}$-discretization.

\subsection{Abstract discrete stationarity system}

Assume that $V_{h} \subset W_{0}^{1, q}(\Omega) \subset W_{0}^{1, q^{\prime}}(\Omega)$ is a discrete space and let $U_{h} \subset L^{2}(\Omega)$ define an (optionally infinite dimensional, i.e. equality is allowed) subspace of $L^{2}(\Omega)$. Let $\pi: L^{2}(\Omega) \rightarrow U_{h}$ be the $L^{2}$-projection from $L^{2}(\Omega)$ onto $U_{h}$ such that the closed convex set

$$
U_{a d, h}^{\pi}:=\left\{v \in U_{h} \mid \pi(a) \leqslant v \leqslant \pi(b)\right\}
$$

is non-empty. We denote the $L^{2}(\Omega)$-projection into $U_{a d, h}^{\pi}$ by $\Pi_{U_{a d, h}^{\pi}}$, write $a_{h}=\pi(a), b_{h}=\pi(b)$, and formulate the discrete version of the optimality system in weak form.

For a discrete adjoint state $p_{h} \in V_{h}$, the corresponding feasible control $u_{h} \in U_{a d, h}^{\pi}$ is defined analogously to (3.4) by

$$
u_{h}=\Pi_{U_{a d, h}^{\pi}}\left(\frac{1}{v} p_{h}\right) .
$$

Furthermore, the variables $\xi_{h}, \lambda_{h} \in V_{h}^{*}$ and $\mu_{h} \in V_{h}$ are given by

$$
\begin{array}{ll}
\forall \psi \in V_{h}: & \left\langle\xi_{h}, \psi\right\rangle_{V_{h}^{*}}=\left\langle A y_{h}, \psi\right\rangle_{W^{-1, q}(\Omega)}-\left(u_{h}+f, \psi\right)_{L^{2}(\Omega)}, \\
\forall \phi \in V_{h}^{*}: & \left\langle\phi, \mu_{h}\right\rangle_{V_{h}^{*}}=-\left\langle\phi, p_{h}\right\rangle_{V_{h}^{*},} \\
\forall \psi \in V_{h}: & \left\langle\lambda_{h}, \psi\right\rangle_{V_{h}^{*}}=\left\langle\psi, A^{*} p_{h}\right\rangle_{W_{0}^{1, q}(\Omega)}+\sum_{w \in I}\left(y_{h}(w)-y_{w}\right) \psi(w) .
\end{array}
$$

Note that we artificially define the Variable $\mu_{h}=-p_{h}$ which can be thought of being a multiplier for the non-negativity condition on $\xi_{h}$. This will be helpful in the derivation of the error representation formula of Theorem 4.2. With the above notation the discrete stationarity system comprises of the conditions (4.1) and (4.2a)-(4.2c) as well as the following complementarity and sign conditions:

$$
\begin{aligned}
y_{h} \geqslant 0, \quad \xi_{h} \geqslant 0, \quad\left\langle\xi_{h}, y_{h}\right\rangle_{V_{h}^{*}} & =0, \\
\forall \psi \in V_{h},\left.\psi\right|_{\left\{y_{h}=0\right\}}=0, \quad\left\langle\lambda_{h}, \psi\right\rangle_{V_{h}^{*}} & =0, \\
\left\langle\xi_{h}, \mu_{h}\right\rangle_{V_{h}^{*}} & =0, \\
\left\langle\lambda_{h}, \mu_{h}\right\rangle_{V_{h}^{*}} & \geqslant 0 .
\end{aligned}
$$

In order to obtain the weighted error representation and in view of (3.5) in Remark 3.3, we replace (4.1) by

$$
\begin{aligned}
& \left(v u_{h}-p_{h}-\sigma_{a h}+\sigma_{b h}, \psi\right)_{L^{2}(\Omega)} & =0, & \\
u_{h}-a_{h} \geqslant 0, & \sigma_{a h} \geqslant 0, & \left(u_{h}-a_{h}, \sigma_{a h}\right)_{L^{2}(\Omega)} & =0, \\
b_{h}-u_{h} \geqslant 0, & \sigma_{b h} \geqslant 0, & \left(b_{h}-u_{h}, \sigma_{b h}\right)_{L^{2}(\Omega)} & =0 .
\end{aligned}
$$


Furthermore, we need to extend the mappings $\xi_{h}$ and $\lambda_{h}$, which are defined as linear mappings on the discrete subspaces only, to the full spaces $W^{-1, q}(\Omega)$ and $W^{-1, q^{\prime}}(\Omega)$, respectively. This (nonunique) extension depends on the concrete choice of discrete spaces and their bases. They are given according to our test scenario in Section 4.3 below.

\subsection{Abstract primal-dual weighted error representation}

In the following we use the abbreviations $x=(y, u, \xi, p)$ and $\Lambda=\left(\lambda, \mu, \sigma_{a}, \sigma_{b}\right)$, and define the MPCC-Lagrangian by

$$
\begin{gathered}
\mathcal{L}(x, \Lambda)=J(y, u)+\langle A y, p\rangle_{W^{-1, q}(\Omega)}-(u+\xi+f, p)_{L^{2}(\Omega)}-\langle\lambda, y\rangle_{W^{-1, q^{\prime}}(\Omega)}-(\xi, \mu)_{L^{2}(\Omega)} \\
-\left(u-a, \sigma_{a}\right)_{L^{2}(\Omega)}-\left(b-u, \sigma_{b}\right)_{L^{2}(\Omega)} .
\end{gathered}
$$

Here we again use $\mu=-p$ as a multiplier for the non-negativity condition on $\xi$. Note that if $\left(x_{h}^{\star}, \Lambda_{h}^{\star}\right)$ solves the discrete stationarity system (4.2), then

$$
\mathcal{L}\left(x_{h}^{\star}, \Lambda_{h}^{\star}\right)=J\left(y_{h}^{\star}, u_{h}^{\star}\right) .
$$

Furthermore, $\mathcal{L}(\cdot, \Lambda)$ is a quadratic functional in $x$, and since its second Fréchet derivative does not depend on $(x, \Lambda)$ we may leave off the argument and abbreviate $\nabla_{x x} \mathcal{L}(x, \Lambda)\left(h_{1}, h_{2}\right)=$ $\nabla_{x x} \mathcal{L}\left(h_{1}, h_{2}\right)$ for $h_{1}, h_{2} \in W_{0}^{1, q}(\Omega) \times L^{2}(\Omega) \times L^{2}(\Omega) \times W_{0}^{1, q^{\prime}}(\Omega)$.

THEOREM 4.1 Assume that $\left(y^{\star}, u^{\star}, \xi^{\star}\right)$ is limiting $\epsilon$-almost C-stationary for (2.6) with associated multipliers $\left(p^{\star}, \lambda^{\star}, \mu^{\star}, \sigma_{a}^{\star}, \sigma_{b}^{\star}\right), \mu=-p \in W_{0}^{1, q^{\prime}}(\Omega)$, and that $\left(y_{h}^{\star}, u_{h}^{\star}, \xi_{h}^{\star}, p_{h}^{\star}, \lambda_{h}^{\star}, \sigma_{a h}^{\star}, \sigma_{b h}^{\star}\right)$ satisfies (4.2). Then it holds that

$$
\begin{aligned}
J\left(y_{h}^{\star}, u_{h}^{\star}\right)-J\left(y^{\star}, u^{\star}\right)= & \frac{1}{2} \nabla_{x x} \mathcal{L}\left(x_{h}^{\star}-x^{\star}, x_{h}^{\star}-x^{\star}\right)+\left\langle\lambda^{\star}, y_{h}^{\star}\right\rangle_{W^{-1, q^{\prime}}(\Omega)}+\left\langle\xi_{h}^{\star}, \mu^{\star}\right\rangle_{W^{-1, q}(\Omega)} \\
& +\left(u_{h}^{\star}-a, \sigma_{a}^{\star}\right)_{L^{2}(\Omega)}+\left(b-u_{h}^{\star}, \sigma_{b}^{\star}\right)_{L^{2}(\Omega)} \\
& +\left(\sigma_{a h}^{\star}, a-a_{h}\right)_{L^{2}(\Omega)}+\left(\sigma_{b h}^{\star}, b_{h}-b\right)_{L^{2}(\Omega)} .
\end{aligned}
$$

Proof. Following the proof of Theorem 3.1 in [27], we use (4.3) and Taylor expansion to obtain

$$
J\left(y_{h}^{\star}, u_{h}^{\star}\right)=\mathcal{L}\left(x^{\star}, \Lambda_{h}^{\star}\right)+\nabla_{x} \mathcal{L}\left(x^{\star}, \Lambda_{h}^{\star}\right)\left(x_{h}^{\star}-x^{\star}\right)+\frac{1}{2} \nabla_{x x} \mathcal{L}\left(x_{h}^{\star}-x^{\star}, x_{h}^{\star}-x^{\star}\right) .
$$

Note that in comparison to [27], where an estimator is derived for a problem class without control constraints and with the state space $H_{0}^{1}(\Omega)$, we now have

$$
\begin{aligned}
& \mathcal{L}\left(x^{\star}, \Lambda_{h}^{\star}\right)=J\left(y^{\star}, u^{\star}\right)-\left\langle\lambda_{h}^{\star}, y^{\star}\right\rangle_{W^{-1, q^{\prime}}(\Omega)}-\left\langle\xi^{\star}, \mu_{h}^{\star}\right\rangle_{W^{-1, q}(\Omega)} \\
& \quad-\left(u^{\star}-a, \sigma_{a h}^{\star}\right)_{L^{2}(\Omega)}-\left(b-u^{\star}, \sigma_{b h}^{\star}\right)_{L^{2}(\Omega)}, \\
& \nabla_{x} \mathcal{L}\left(x^{\star}, \Lambda_{h}^{\star}\right)\left(x_{h}^{\star}-x^{\star}\right)=\left\langle\lambda_{h}^{\star}, y^{\star}\right\rangle_{W^{-1, q^{\prime}}(\Omega)}+\left\langle\lambda^{\star}, y_{h}^{\star}\right\rangle_{W^{-1, q^{\prime}}(\Omega)} \\
& \quad+\left\langle\xi^{\star}, \mu_{h}^{\star}\right\rangle_{W^{-1, q}(\Omega)}+\left\langle\xi_{h}^{\star}, \mu^{\star}\right\rangle_{W^{-1, q}(\Omega)} \\
& \quad+\left(u^{\star}-a_{h}, \sigma_{a h}^{\star}\right)_{L^{2}(\Omega)}+\left(u_{h}^{\star}-a, \sigma_{a}^{\star}\right)_{L^{2}(\Omega)}+\left(b_{h}-u^{\star}, \sigma_{b h}^{\star}\right)_{L^{2}(\Omega)}+\left(b-u_{h}^{\star}, \sigma_{b}^{\star}\right)_{L^{2}(\Omega)} .
\end{aligned}
$$

Summing these terms yields the assertion. 
THEOREM 4.2 Under the assumptions of Theorem 4.1, let $\delta x_{h}=\left(\delta y_{h}, \delta u_{h}, \delta \xi_{h}, \delta p_{h}\right) \in V_{h} \times U_{h} \times$ $V_{h}^{*} \times V_{h}$. Then it holds that

$$
\begin{aligned}
& 2\left(J\left(y^{\star}, u^{\star}\right)-J\left(y_{h}^{\star}, u_{h}^{\star}\right)\right)= \\
& \left\langle A y_{h}^{\star}, p^{\star}-\delta p_{h}\right\rangle_{W^{-1, q}(\Omega)}-\left(u_{h}^{\star}+f, p^{\star}-\delta p_{h}\right)_{L^{2}(\Omega)}-\left\langle\xi_{h}^{\star}, p^{\star}-\delta p_{h}\right\rangle_{W^{-1, q}(\Omega)} \\
& +\left\langle y^{\star}-\delta y_{h}, A^{*} p_{h}^{\star}\right\rangle_{W_{0}^{1, q}(\Omega)}+\sum_{w \in I}\left(y_{h}^{\star}-y_{w}\right)\left(y^{\star}-\delta y_{h}\right)(w)-\left\langle\lambda_{h}^{\star}, y^{\star}-\delta y_{h}\right\rangle_{W^{-1, q^{\prime}}(\Omega)} \\
& +\left(v u_{h}^{\star}-p_{h}^{\star}-\sigma_{a h}^{\star}+\sigma_{b h}^{\star}, u^{\star}-\delta u_{h}\right)_{L^{2}(\Omega)} \\
& +\left\langle\xi^{\star}-\delta \xi_{h},-\mu_{h}^{\star}-p_{h}^{\star}\right\rangle_{W^{-1, q}(\Omega)} \\
& +\left\langle\lambda_{h}^{\star}, y^{\star}\right\rangle_{W^{-1, q^{\prime}}(\Omega)}-\left\langle\xi_{h}^{\star}, \mu^{\star}\right\rangle_{W^{-1, q}(\Omega)}-\left\langle\lambda^{\star}, y_{h}^{\star}\right\rangle_{W^{-1, q^{\prime}}(\Omega)}+\left\langle\xi^{\star}, \mu_{h}^{\star}\right\rangle_{W^{-1, q}(\Omega)} \\
& -\left(u_{h}^{\star}-a, \sigma_{a}^{\star}\right)_{L^{2}(\Omega)}+\left(u^{\star}-a_{h}, \sigma_{a h}^{\star}\right)_{L^{2}(\Omega)} \\
& -\left(b-u_{h}^{\star}, \sigma_{b}^{\star}\right)_{L^{2}(\Omega)}+\left(b_{h}-u^{\star}, \sigma_{b h}^{\star}\right)_{L^{2}(\Omega)} \\
& -\left(a-a_{h}, \sigma_{a h}^{\star}\right)_{L^{2}(\Omega)}-\left(b_{h}-b, \sigma_{b h}^{\star}\right)_{L^{2}(\Omega)} .
\end{aligned}
$$

Proof. We take Theorem 4.1 as a starting point and compute an explicit representation of $\nabla_{x x} \mathcal{L}\left(x_{h}^{\star}-x^{\star}, x_{h}^{\star}-x^{\star}\right)$. Taylor expansion of the gradient $\nabla_{x} \mathcal{L}\left(x_{h}^{\star}, \Lambda_{h}^{\star}\right)$ in the direction $\left(x^{\star}-x_{h}^{\star}\right)$ at $x^{\star}$ yields that

$$
\nabla_{x} \mathcal{L}\left(x_{h}^{\star}, \Lambda_{h}^{\star}\right)\left(x_{h}^{\star}-x^{\star}\right)=\nabla_{x} \mathcal{L}\left(x^{\star}, \Lambda_{h}^{\star}\right)\left(x_{h}^{\star}-x^{\star}\right)+\nabla_{x x} \mathcal{L}\left(x_{h}^{\star}-x^{\star}, x_{h}^{\star}-x^{\star}\right) .
$$

Note that the Lagrangian is quadratic and thus higher order derivatives vanish. We can recycle the gradient from the proof of Theorem 4.1 to obtain

$$
\begin{aligned}
\nabla_{x x} \mathcal{L} & \left(x_{h}^{\star}-x^{\star}, x_{h}^{\star}-x^{\star}\right)=\nabla_{x} \mathcal{L}\left(x_{h}^{\star}, \Lambda_{h}^{\star}\right)\left(x_{h}^{\star}-x^{\star}\right)-\left\langle\lambda_{h}^{\star}, y^{\star}\right\rangle_{W^{-1, q^{\prime}}(\Omega)}-\left\langle\lambda^{\star}, y_{h}^{\star}\right\rangle_{W^{-1, q^{\prime}}(\Omega)} \\
& -\left\langle\xi^{\star}, \mu_{h}^{\star}\right\rangle_{W^{-1, q}(\Omega)}-\left\langle\xi_{h}^{\star}, \mu^{\star}\right\rangle_{W^{-1, q}(\Omega)}-\left(u^{\star}-a_{h}, \sigma_{a h}^{\star}\right)_{L^{2}(\Omega)}-\left(u_{h}^{\star}-a, \sigma_{a}^{\star}\right)_{L^{2}(\Omega)} \\
& -\left(b_{h}-u^{\star}, \sigma_{b h}^{\star}\right)_{L^{2}(\Omega)}-\left(b-u_{h}^{\star}, \sigma_{b}^{\star}\right)_{L^{2}(\Omega)} .
\end{aligned}
$$

Employing the discrete stationarity system (4.2), we replace the term $\nabla_{x} \mathcal{L}\left(x_{h}^{\star}, \Lambda_{h}^{\star}\right)\left(x_{h}^{\star}-x^{\star}\right)$ by $-\nabla_{x} \mathcal{L}\left(x_{h}^{\star}, \Lambda_{h}^{\star}\right)\left(x^{\star}-\delta x_{h}\right)$ and evaluate this term to obtain (4.4a)-(4.4d). The remaining terms sum up to give lines (4.4e)-(4.4h).

REMARK 4.3 Since the mapping $\pi$ for the control constraints satisfies $(\pi(\psi), \sigma)_{L^{2}(\Omega)}=$ $(\psi, \sigma)_{L^{2}(\Omega)}$ for all $\psi \in L^{2}(\Omega)$ and $\sigma \in U_{h}$, the terms (4.4f)-(4.4h) resolve to

$$
-\left(u_{h}^{\star}-a, \sigma_{a}^{\star}\right)_{L^{2}(\Omega)}+\left(u^{\star}-a, \sigma_{a h}^{\star}\right)_{L^{2}(\Omega)}-\left(b-u_{h}^{\star}, \sigma_{b}^{\star}\right)_{L^{2}(\Omega)}+\left(b-u^{\star}, \sigma_{b h}^{\star}\right)_{L^{2}(\Omega)} .
$$

REMARK 4.4 In the case that $U_{a d}=L^{2}(\Omega)$, an abstract error representation formula is obtained by omitting the terms (4.4f)-(4.4h) and setting $\sigma_{a h}^{\star}=\sigma_{b h}^{\star}=0$ in (4.4c).

\section{3 $P^{1}$ finite element discretization}

In this section we fix some more details of the discretization. For a polygonal bounded domain $\Omega$ with triangulation $\mathcal{J}$ that is regular in the sense of [2, p. 394], we consider exemplarily $P_{0}^{1}(\mathcal{J})$, the 
piecewise affine and globally continuous finite elements with zero boundary conditions. We denote the set of all interior nodes by $\eta$, the set of all edges by $\varepsilon$ and, for $\omega \subset \Omega$, the set of edges $E \subset \omega$ by $\mathcal{\varepsilon}(\omega)$. For an inner edge $E=T^{+} \cap T^{-} \in \mathcal{E}$ and triangles $T^{+}, T^{-} \in \mathcal{J}$ with outer unit normal vector $v_{T^{+}, E}=-v_{T^{-}, E}$, we define the edge jump of a piecewisely defined function $v_{h}$ over $E$ by

$$
\left[\nabla v_{h}\right]_{E}:=\left(\left.\nabla v_{h}\right|_{T^{+}}-\left.\nabla v_{h}\right|_{T^{-}}\right) \cdot v_{T^{+}, E}=\left(\left.\nabla v_{h}\right|_{T^{-}}-\left.\nabla v_{h}\right|_{T^{+}}\right) \cdot v_{T^{-}, E} .
$$

If $E \subset \partial \Omega$ we define $\left[\nabla v_{h}\right]_{E}:=0$. For $A=-\Delta$, integration by parts yields for the bilinear form $a$ from Remark 2.2 and $z \in W^{1, q^{\prime}}(\Omega)$ that

$$
\left\langle A v_{h}, z\right\rangle_{W^{-1, q}(\Omega)}=\mathfrak{a}\left(v_{h}, z\right)=\sum_{T \in \mathcal{T}} \sum_{E \in \mathcal{E}(T)} \frac{1}{2} \int_{E}\left[\nabla v_{h}\right]_{E} z d S .
$$

Note that if $v_{h} \in P_{0}^{1}(\mathcal{J})$ is in fact piecewise affine, then $\left.\nabla v_{h}\right|_{T}$ is constant and thus

$$
\int_{E}\left[\nabla v_{h}\right]_{E} z d S=\left[\nabla v_{h}\right]_{E} \int_{E} z d S .
$$

We set $V_{h}=P_{0}^{1}(\mathcal{J})$ and $U_{h}=L^{2}(\Omega)$, such that $\pi=\left.\mathrm{id}\right|_{L^{2}(\Omega)}$ and $U_{a d}^{h}=U_{a d}$. Furthermore, we denote the canonical nodal basis of $P_{0}^{1}(\mathcal{J})$ by $\left\{\varphi_{z} \mid z \in \eta\right\}$. The extensions of $\xi_{h}$ and $\lambda_{h}$ to $W^{-1, q^{\prime}}(\Omega)$, which are only defined in (4.2a)-(4.2c) on the discrete subspace $V_{h}$, are given as follows. Respecting the multiplier character of $\xi_{h}$ and $\lambda_{h}$, we set

$$
\xi_{h}=\sum_{z \in \mathfrak{n}} \xi_{h, z} \delta_{z} \in W^{-1, q^{\prime}}(\Omega) \quad \text { and } \quad \lambda_{h}=\sum_{z \in \mathfrak{n}} \lambda_{h, z} \delta_{z} \in W^{-1, q^{\prime}}(\Omega),
$$

where for $z \in \eta, \xi_{h, z}=\left\langle\xi_{h}, \varphi_{z}\right\rangle_{W^{-1, q}(\Omega)}$ and $\lambda_{h, z}=\left\langle\lambda_{h}, \varphi_{z}\right\rangle_{W^{-1, q^{\prime}}(\Omega)}$. The bounded linear functional $\xi_{h}: W_{0}^{1, q}(\Omega) \rightarrow \mathbb{R}$ can be further extended to $W_{0}^{1, q^{\prime}}(\Omega)$. However, in our test setting, we do not explicitly use this extension because $p_{h} \in V_{h} \subset W_{0}^{1, q}(\Omega) \hookrightarrow W_{0}^{1, q^{\prime}}(\Omega)$, and thus do not give an explicit definition here. We follow the discussion in [23] and write pairings of $\xi_{h}$ and $\lambda_{h}$ with arbitrary functions in $W_{0}^{1, q}(\Omega)$ as a sum over the triangles in $\mathcal{J}$ : First, define numbers $N_{z}=\operatorname{card}(\{T \in \mathcal{J} \mid z \in \eta(T)\})$ of triangles intersecting in nodes $z \in \eta$. Then, for $v \in P_{0}^{1}(\mathcal{J})$ we can localize the dual pairing $\left\langle\xi_{h}, v\right\rangle_{W^{-1, q^{\prime}}(\Omega)}$ (and $\left\langle\lambda_{h}, \bar{v}\right\rangle_{W^{-1, q^{\prime}}(\Omega)}$ analogously) as follows:

$$
\left\langle\xi_{h}, v\right\rangle_{W^{-1, q^{\prime}}(\Omega)}=\sum_{z \in \mathfrak{n}} \xi_{h, z}\left\langle\delta_{z}, v\right\rangle_{W^{-1, q^{\prime}}(\Omega)}=\sum_{T \in \mathcal{T}} \sum_{z \in \cap(T)} \frac{1}{N_{z}} \xi_{h, z} v(z) .
$$

The dual variable $\mu_{h}$ corresponding to the non-negativity constraint on $\xi_{h}$ is set to $\mu_{h}=$ $-p_{h} \in P_{0}^{1}(\mathcal{J})$. Then condition (4.2b) is satisfied and (4.4d) in the error representation formula of Theorem 4.2 vanishes.

We suggest a heuristic way to replace the continuous solutions which typically arise in goal oriented dual weighted error estimation as follows. On every triangle $T \in \mathcal{T}$, we replace a discrete function $v_{h} \in P_{0}^{1}(\mathcal{J})$ by a quadratic function, which we denote by $\left.\tilde{v}_{h}\right|_{T}$. We compute $\left.\tilde{v}_{h}\right|_{T}$ such that it minimizes the least squares distance to the values of $v_{h}$ in the midpoints of the edges of $T$ and in the nodes of its neighboring elements sharing an edge with $T$ which are not nodes of $T$, see Figure 1. If this minimization problem has more than one solution, we choose the one with minimal $\ell_{2}$ norm of the coefficient vector of the polynomial in the canonical basis $\left\{1, x_{1}, x_{2}, x_{1}^{2}, x_{1} x_{2}, x_{2}^{2}\right\}$. These $\mathcal{J}$-piecewisely defined quadratic functions are assembled to an $L^{2}$-function $\tilde{v}_{h}$ on $\Omega$. 

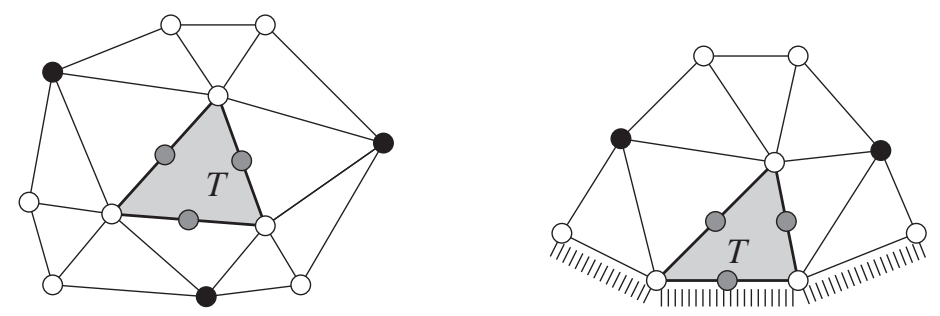

FIG. 1. The $P_{2}$ approximation of a discrete function over an element $T$ (gray shaded) is defined via the least squares minimization problem of its values at the mid points of the edges of $T$ (gray circles) and the nodes of its (up to) three neighboring elements which are not nodes of $T$ (black circles).

\subsection{Primal-dual weighted error indicator for $P^{1}$ finite elements}

With the notation of Section 4.3 at hand, we derive an a posteriori error indicator from Theorem 4.2 with $\delta x_{h}=x_{h}^{\star}$ and $x^{\star}$ replaced by $\tilde{x}_{h}^{\star}$. The indicator

$$
\eta=\sum_{T \in \mathcal{T}} \eta_{T}
$$

contains the error contribution $\eta_{T}$ on each triangle $T \in \mathcal{J}$ and is composed of different parts belonging to the lines in the error representation formula (4.4), $\eta_{T}=\eta_{\mathrm{PDE} 1, T}+\eta_{\mathrm{PDE} 2, T}+\eta_{\mathrm{CM}, T}+$ $\eta_{\mathrm{U}, T}$. To keep notation simple, we consider only the case $A=-\Delta$ and $\mathfrak{a}(\cdot, \cdot)=\langle A \cdot, \cdot\rangle_{W^{-1, q}(\Omega)}=$ $\langle\nabla \cdot, \nabla \cdot\rangle_{L^{q}(\Omega)}$. In the rest of this section we discuss the local error contributions $\eta_{\mathrm{PDE} 1, T}, \eta_{\mathrm{PDE} 2, T}$, $\eta_{\mathrm{CM}, T}$ and $\eta_{\mathrm{U}, T}$.

We start with the part of the indicator corresponding to the dual weighted primal partial differential equation residual (4.4a). Making use of the edge jump formula (4.5) and the localization of $\xi_{h}^{\star}$ from (4.6), we rearrange these terms as a sum of integrals over the elements $T \in \mathcal{J}$. In this way, we obtain a localized version of (4.4a) which still contains the exact dual solution $p^{\star}$. In order to get a computable estimation for the local error indicators, we replace $p^{\star}$ by $\tilde{p}_{h}^{\star}$ and obtain $\eta_{\mathrm{PDE} 1}=\sum_{T \in \mathcal{J}} \eta_{\mathrm{PDE} 1, T}$, where

$$
\begin{aligned}
\eta_{\mathrm{PDE} 1, T}:=\frac{1}{2} \mid \int_{\partial T} \frac{1}{2}\left[\nabla y_{h}^{\star}\right]_{\partial T}\left(\tilde{p}_{h}^{\star}-p_{h}^{\star}\right) d S-\int_{T}\left(u_{h}^{\star}\right. & +f)\left(\tilde{p}_{h}^{\star}-p_{h}^{\star}\right) d x \\
& -\sum_{z \in \eta(T)} \frac{1}{N_{z}} \xi_{h}^{\star}\left(\tilde{p}_{h}^{\star}(z)-p_{h}^{\star}(z)\right) \mid .
\end{aligned}
$$

Note that due to the low regularity of the adjoint state, there is no evidence that $\tilde{p}_{h}^{\star}$ yields a better approximation of $p^{\star}$ than $p_{h}^{\star}$. The lack of regularity comes from the spiky structure of $p^{\star}$ in the tracking points. In regions away from those points, the adjoint state may admit locally a higher regularity. In this case, $P^{2}$ interpolants yield advantages over the affine approximation.

For line (4.4b), which corresponds to the primal weighted adjoint equation, we again use (4.5) as well as formula (4.6) for the multiplier $\lambda_{h}^{\star}$. Furthermore, the set of tracking points $I \subset \Omega$ is split into those in the interior of triangles, those on edges but not nodes of triangles and those that are in fact nodes of triangles. Then a sum $\sum_{w \in I} \psi(w)$ is split into its contribution on individual elements 
$T \in \mathcal{J}$ as follows:

$$
\sum_{w \in I} \psi(w)=\sum_{T \in \mathcal{T}}\left(\sum_{w \in I \cap \operatorname{int}(T)} \psi(w)+\frac{1}{2} \sum_{w \in I \cap \partial T \backslash n} \psi(w)+\sum_{w \in I \cap n} \frac{1}{N_{w}} \psi(w)\right) .
$$

Setting $\psi=\left(y_{h}^{\star}-y_{w}\right)\left(\tilde{y}_{h}^{\star}-y_{h}^{\star}\right)$ and replacing the exact solution $y^{\star}$ by its $P^{2}$-approximation $\tilde{y}_{h}^{\star}$ one obtains

$$
\begin{aligned}
\eta_{\mathrm{PDE} 2, T}:=\frac{1}{2} \mid \int_{\partial T} & \frac{1}{2}\left[\nabla p_{h}^{\star}\right]_{\partial T}\left(\tilde{y}_{h}^{\star}-y_{h}^{\star}\right) d S-\sum_{z \in \cap(T)} \frac{1}{N_{z}} \lambda_{h z}^{\star}\left(\tilde{y}_{h}^{\star}(z)-y_{h}^{\star}(z)\right) \\
& +\sum_{w \in I \cap \operatorname{int}(T)}\left(y_{h}^{\star}(w)-y_{w}\right)\left(\tilde{y}_{h}^{\star}(w)-y_{h}^{\star}(w)\right) \\
& +\frac{1}{2} \sum_{w \in I \cap \partial T \backslash \eta}\left(y_{h}^{\star}(w)-y_{w}\right)\left(\tilde{y}_{h}^{\star}(w)-y_{h}^{\star}(w)\right) \\
& +\sum_{w \in I \cap \eta} \frac{1}{N_{w}}\left(y_{h}^{\star}(w)-y_{w}\right)\left(\tilde{y}_{h}^{\star}(w)-y_{h}^{\star}(w)\right) \mid .
\end{aligned}
$$

The expressions in (4.4c) and (4.4d) yield zero due to the definitions of $\sigma_{a h}^{\star}, \sigma_{b h}^{\star}$ (see (4.2h) with $\left.U_{h}=L^{2}(\Omega)\right)$ and $\mu_{h}^{\star}$.

The terms in (4.4e) reflecting the mismatch in complementarity of $y_{h}^{\star}, \lambda_{h}^{\star}, \xi_{h}^{\star}, \mu_{h}^{\star}=-p_{h}^{\star}$ and their continuous counterparts are split into two different types of duality pairings. In fact, we have the pairings of discrete multipliers and continuous solutions versus those containing multipliers from the continuous problem, which are hard to approximate. Respecting the different structure, we handle the first two products differently to the third and forth. Owing to the discrete complementarity conditions (4.2e) and (4.2f), and replacing $\mu^{\star}, \mu_{h}^{\star}$ by $-p^{\star},-p_{h}^{\star}$ it holds that

$$
\begin{aligned}
\left\langle\lambda_{h}^{\star}, y^{\star}\right\rangle_{W^{-1, q^{\prime}}(\Omega)} & =\left\langle\lambda_{h}^{\star}, y^{\star}-y_{h}^{\star}\right\rangle_{W^{-1, q^{\prime}}(\Omega)}, \\
-\left\langle\xi_{h}^{\star}, \mu^{\star}\right\rangle_{W^{-1, q}(\Omega)} & =\left\langle\xi_{h}^{\star}, p^{\star}-p_{h}^{\star}\right\rangle_{W^{-1, q}(\Omega)} .
\end{aligned}
$$

Analogously to the above, we replace $y^{\star}$ and $p^{\star}$ by $\tilde{y}_{h}^{\star}$ and $\tilde{p}_{h}^{\star}$, respectively and obtain a posteriori terms, which can again be localized using (4.6). This yields the first line in (4.14) below. The multipliers $\lambda^{\star}$ and $\xi^{\star}$ in the remaining products are eliminated by using the dual and primal equations (3.6b) and (2.3), respectively. Then, with the complementarity relations (3.6c) and (3.6f), it holds that

$$
\begin{aligned}
\left\langle\lambda^{\star}, y_{h}^{\star}\right\rangle_{W^{-1, q^{\prime}}(\Omega)} & =\left\langle\lambda^{\star}, y_{h}^{\star}-y^{\star}\right\rangle_{W^{-1, q^{\prime}}(\Omega)} \\
& =\left\langle A\left(y_{h}^{\star}-y^{\star}\right), p^{\star}\right\rangle_{W^{-1, q}(\Omega)}-\sum_{w \in I}\left(\left(y^{\star}-y_{w}\right)\left(y_{h}^{\star}-y^{\star}\right)\right)(w)
\end{aligned}
$$

and

$$
\begin{aligned}
\left\langle\xi^{\star}, \mu_{h}^{\star}\right\rangle_{W^{-1, q}(\Omega)} & =\left\langle\xi^{\star}, p_{h}^{\star}-p^{\star}\right\rangle_{W^{-1, q}(\Omega)} \\
& =\left\langle A y^{\star}, p_{h}^{\star}-p^{\star}\right\rangle_{W^{-1, q}(\Omega)}-\left(u^{\star}+f, p_{h}^{\star}-p^{\star}\right)_{L^{2}(\Omega)}
\end{aligned}
$$


We now replace $y^{\star}$ by $\tilde{y}_{h}^{\star}$ and $p^{\star}$ by $\tilde{p}_{h}^{\star}$ in (4.10)-(4.13), split the terms as described above, and use (4.5) to obtain

$$
\begin{aligned}
& \eta_{\mathrm{CM}, T}:=\frac{1}{2}\left|\sum_{z \in \mathfrak{n}(T)} \frac{1}{N_{z}} \lambda_{h z}^{\star}\left(\tilde{y}_{h}^{\star}(z)-y_{h}^{\star}(z)\right)\right|+\frac{1}{2}\left|\sum_{z \in n_{(T)}} \frac{1}{N_{z}} \xi_{h z}^{\star}\left(\tilde{p}_{h}^{\star}(z)-p_{h}^{\star}(z)\right)\right| \\
& +\frac{1}{2} \mid \int_{\partial T} \frac{1}{2}\left[\nabla \tilde{p}_{h}^{\star}\right]_{\partial T}\left(\tilde{y}_{h}^{\star}-y_{h}^{\star}\right) d S+\sum_{w \in I \cap \operatorname{int}(T)}\left(\tilde{y}_{h}^{\star}(w)-y_{w}\right)\left(\tilde{y}_{h}^{\star}(w)-y_{h}^{\star}(w)\right) \\
& +\frac{1}{2} \sum_{w \in I \cap \partial T \backslash n}\left(\tilde{y}_{h}^{\star}(w)-y_{w}\right)\left(\tilde{y}_{h}^{\star}(w)-y_{h}^{\star}(w)\right) \\
& +\sum_{w \in I \cap n} \frac{1}{N_{w}}\left(\tilde{y}_{h}^{\star}(w)-y_{w}\right)\left(\tilde{y}_{h}^{\star}(w)-y_{h}^{\star}(w)\right) \mid \\
& +\frac{1}{2}\left|\int_{\partial T} \frac{1}{2}\left[\nabla \tilde{y}_{h}^{\star}\right]_{\partial T}\left(\tilde{p}_{h}^{\star}-p_{h}^{\star}\right) d S-\int_{T}\left(\tilde{u}_{h}^{\star}+f\right)\left(\tilde{p}_{h}^{\star}-p_{h}^{\star}\right) d x\right| .
\end{aligned}
$$

Since we have the situation of Remark 4.3, the terms (4.4f)-(4.4h) resolve to

$$
\begin{aligned}
\eta_{\mathrm{U}, T}:=\frac{1}{2}\left|\int_{T}\left(u_{h}^{\star}-a\right) \sigma_{a}^{\star} d x\right|+\frac{1}{2} \mid & \int_{T}\left(\tilde{u}_{h}^{\star}-a\right) \sigma_{a h}^{\star} d x \mid \\
& +\frac{1}{2}\left|\int_{T}\left(u_{h}^{\star}-b\right) \sigma_{b}^{\star} d x\right|+\frac{1}{2}\left|\int_{T}\left(\tilde{u}_{h}^{\star}-b\right) \sigma_{b h}^{\star} d x\right| .
\end{aligned}
$$

Note that we have replaced $u^{\star}$ by $\tilde{u}_{h}^{\star}$, but there is no assertion on the approximation quality of the $P^{2}$-interpolant in comparison with the $P^{1}$-solution.

Altogether the definitions in (4.8), (4.9), (4.14), (4.15) yield a fully a posteriori and local indicator (4.7) for the difference $J\left(y^{\star}, u^{\star}\right)-J\left(y_{h}^{\star}, u_{h}^{\star}\right)$.

\section{Numerical realization}

We start in Section 5.1 with a brief overview of the adaptive finite element method (AFEM) and its subroutines in Section 5.1 before establishing an optimization algorithm which works analogously to the analysis. In Section 5.2 we describe the Newton-type method used to solve the discretized stationarity system corresponding to (3.2), and the penalized smoothed state equation (2.17c) for fixed parameters $\gamma$ and $\epsilon$. Taking these parameters to infinity and to zero, respectively, a solution to the discrete C-stationarity system (4.2) can be obtained. We end this paper by discussing numerical results which are contained in Section 5.3.

\subsection{Adaptive finite element scheme}

The mesh-adaption process in a typical AFEM loop is guided iteratively by local error indicators based on solutions of the problem on the current mesh. Elements with large local error indicators are marked for refinement, and a superset of the marked elements is actually refined to maintain 
a regular mesh. The algorithm, which is stated in Algorithm 1 repeats the steps solveMPEC estimate - mark - refine until a given complexity $N$ (e.g. the number of degrees of freedom $\operatorname{nrDOF}\left(\mathcal{J}_{h}\right)$ in the SOLVE procedure) is reached.

We indicate the AFEM level by $h$. Step 2 of Algorithm 1 (subroutine solveMPEC) is described in detail in Section 5.2. In this section we discuss briefly steps 3, 7 and 8.

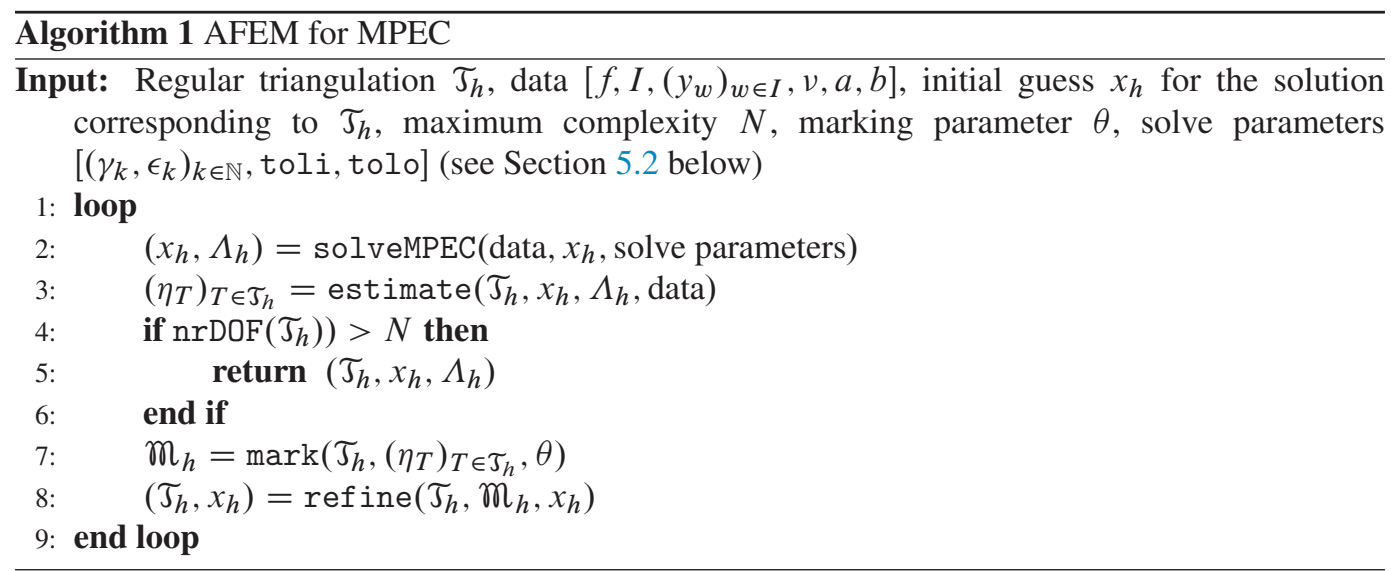

The calculation of the local error indicator

$$
\eta_{h}=\sum_{T \in \mathcal{T}} \eta_{T}=\sum_{T \in \mathcal{T}} \eta_{\mathrm{PDE} 1, T}+\eta_{\mathrm{PDE} 2, T}+\eta_{\mathrm{CM}, T}+\eta_{\mathrm{U}, T}
$$

from the discrete solutions on the current mesh $\mathcal{T}_{h}$ (subroutine estimate in step 3) follows from Section 4.4. The integrals over triangles and edges are computed using Gauss quadrature rules which are exact for polynomial data.

To obtain the next AFEM level, we use a bulk criterion (subroutine mark) to define the set $m_{h} \subset \mathcal{J}_{h}$ of triangles marked for refinement. More precisely, in step 7 we take the set $m_{h} \subset \mathcal{J}_{h}$ of minimal cardinality such that for a bulk parameter $\theta \in(0,1)$ it holds that

$$
\theta \eta_{h} \leqslant \sum_{T \in m_{h}} \eta_{T}
$$

In the refinement step (subroutine refine in step 8) one has to avoid hanging nodes and guarantee shape regularity of the triangulation. This is achieved by a simple bisection rule from the grid manager AluGrid; see [5, 16]. The refinement routine also generates a starting point for the iterative optimization algorithm at the next AFEM level by prolongating the current solution $\left(x_{h}, \Lambda_{h}\right)$ to the refined mesh.

\subsection{Solver for the discretized C-stationarity system}

On a fixed AFEM-level $h$, we solve the discrete C-stationarity system (4.2) according to the penalization scheme from Section 2. This requires an inner and an outer loop: The inner loop solves the discrete stationarity system for a penalized problem with fixed penalization and smoothing 
parameters $\gamma, \epsilon(\gamma), r>0$, whereas the outer loop controls residuals and adjusts the parameters until some break criterion is satisfied.

Concerning the inner loop, we first eliminate the control $u_{h}$ utilizing (4.1), i.e., for $x \in \Omega$, we set

$$
u_{h}(x)=\left\{\begin{array}{cll}
a(x) & \text { if } & \frac{1}{v} p_{h}(x)<a(x), \\
\frac{1}{v} p_{h}(x) & \text { if } & a(x) \leqslant \frac{1}{v} p_{h}(x) \leqslant b(x), \\
b(x) & \text { if } & b(x)<\frac{1}{v} p_{h}(x) .
\end{array}\right.
$$

The discretization of the penalized smoothed stationarity system then reads: For all $\psi \in V_{h}$,

$$
\begin{array}{r}
\langle A y, \psi\rangle_{H^{-1}(\Omega)}-\gamma_{k} \int_{\Omega}\left(\max _{\epsilon_{k}}(0,-y)+\Pi_{U_{a d, h}^{\pi}}\left(\frac{1}{\nu} p\right)+f\right) \psi d x=0, \\
\left\langle\psi, A^{*} p\right\rangle_{H_{0}^{1}(\Omega)}+\gamma_{k} \int_{\Omega} \max _{\epsilon_{k}}^{\prime}(0,-y) p \psi d x+\sum_{w \in I} \frac{1}{\left|B_{r_{k}}(w)\right|} \int_{B_{r_{k}}(w)}\left(y-y_{w}\right) \psi d x=0 .
\end{array}
$$

To approximate the averaged integrals over $B_{r_{k}}(w)$ for $w \in I \subset \Omega$ and $r_{k} \rightarrow 0$ we simply evaluate the integrand in $w$. A tuple $\left(y_{k}, p_{k}\right) \in P_{0}^{1}(\mathcal{J}) \times P_{0}^{1}(\mathcal{J})$ is thus a solution of the discretized penalized smoothed optimal control problem if it is a zero of the mapping $F_{k}: P_{0}^{1}(\tau) \times P_{0}^{1}(\mathcal{J}) \rightarrow \mathbb{R}^{2 \operatorname{card}(\eta)}$ with

$$
\begin{aligned}
& F_{k}(y, p) \\
& =\left(\begin{array}{c}
\left\langle A y, \varphi_{z}\right\rangle_{H^{-1}(\Omega)}-\gamma_{k} \int_{\Omega}\left(\max _{\epsilon_{k}}(0,-y)+\Pi_{U_{a d, h}^{\pi}}\left(\frac{1}{\nu} p\right)+f\right) \varphi_{z} d x \\
\left\langle\varphi_{z}, A^{*} p\right\rangle_{H_{0}^{1}(\Omega)}+\gamma_{k} \int_{\Omega} \max _{\epsilon_{k}}^{\prime}(0,-y) p \varphi_{z} d x+\sum_{w \in I}\left(y(w)-y_{w}\right) \varphi_{z}(w)
\end{array}\right)_{z \in \cap} .
\end{aligned}
$$

Note that in a function space context, the image space of $F$ has to be understood as a subspace of $H^{-1}(\Omega)$. We compute the state $y_{k}$ and the adjoint state $p_{k}$ such that $F_{k}\left(y_{k}, p_{k}\right)=0$. Numerically we terminate the root finding iteration for (5.2) (the inner loop) when $\left\|F_{k}(y, p)\right\|_{H^{-1}(\Omega)} \leqslant$ toli.

In the outer loop we set

$$
\begin{aligned}
& \sigma_{a k}=\left(a-\frac{1}{v} p_{k}\right)_{+}, \quad \sigma_{b k}=\left(\frac{1}{v} p_{k}-b\right)_{+}, \quad u_{k}=\frac{1}{v} p_{k}+\sigma_{a k}-\sigma_{b k}, \\
& \xi_{k}=\gamma_{k} \max _{\epsilon_{k}}\left(0,-y_{k}\right), \quad \lambda_{k}=-\gamma_{k} \max _{\epsilon_{k}}^{\prime}\left(0,-y_{k}\right) p_{k}, \quad \mu_{k}=-p_{k} .
\end{aligned}
$$

With these choices, the equations (4.2a), (4.2b), (4.2c), (4.2h) and the sign conditions in (4.2i) and (4.2j) are generically satisfied. We compute the sum of the norms of the remaining residuals of the discrete C-stationarity system (4.2) as $r_{k}=r_{1}+r_{2}+r_{3}+r_{4}$, where

$$
\begin{array}{ll}
r_{1}=\left|\left\langle\xi_{k}, y_{k}\right\rangle_{V_{h}^{*}}\right|+\sum_{z \in \mathfrak{n}}\left(-\left\langle\xi_{k}, \varphi_{z}\right\rangle_{V_{h}^{*}}\right)_{+}+\left\|\left(-y_{k}\right)_{+}\right\|_{L^{2}(\Omega)}, & r_{2}=\left|\left\langle\lambda_{k}, y_{k}\right\rangle_{V_{h}^{*}}\right|, \\
r_{3}=\left|\left\langle\xi_{k}, p_{k}\right\rangle_{V_{h}^{*}}\right|, & r_{4}=\left(-\left\langle\lambda_{k}, \mu_{k}\right\rangle_{V_{h}^{*}}\right)_{+} .
\end{array}
$$


The outer loop breaks, when $r_{k}<$ tolo. Then, the discrete solutions are returned to the AFEM loop, the discrete data is refined, and a new outer loop is initialized. Note that here we use the value of $\gamma$ in the step before refinement as initial value for the outer loop on the refined mesh. If the residual $r_{k}$ is not sufficiently small, then the parameters $\gamma_{k}$ and $\epsilon_{k}$ are adjusted and a new inner loop is initialized.

\subsection{Numerical results}

Finally, we present numerical results obtained by applying our AFEM approach to two examples. We implemented our method in DUNE (Distributed and Unified Numerics Environment), see [8, 9, $12,17]$ and also the web pages [7, 18].

The parameters and update rules appearing in Algorithm 1 are set to the same values in both examples. In particular, the marking parameter in Algorithm 1 is set to $\theta=0.5$. In the first example, we additionally tested the algorithm for different values of $\theta$, cf. Figure 6 . We start with the penalty parameter $\gamma_{0}=100$, and update it according to $\gamma_{k+1}=\gamma_{k} \cdot 1.2$. The smoothing parameter is computed as $\epsilon_{k}=\epsilon\left(\gamma_{k}\right)=0.1 \cdot \gamma_{k}^{-1.2}$ so the product $\epsilon_{k} \gamma_{k}$ converges to zero as $k \rightarrow \infty$. The tolerances for the residuals in the inner and outer loop are set to toli $=10^{-6}$ and tolo $=10^{-5}$. Note that toli should not be larger than tolo. In the case of equality it might be a matter of a constant in the estimate that ensures the limiting process. In order to avoid a discussion of this constant we decided to choose the tolerances such that the smoothed problem is solved slightly more accurately than the MPEC.

EXAMPLE 5.1 The first example takes place on the square domain $\Omega=(0,1)^{2}$. The set of tracking points is

$$
I=\{(0.125,0.125),(0.125,0.5),(0.375,0.375),(0.5,0.125)\},
$$

and $y_{w}=1$ for $w=(0.125,0.125), y_{w}=0$ otherwise. We set the parameter for the $L^{2}$-averaged cost of the control to be $v=0.003$, set $f \equiv 0$ (i.e., except for the control action, there is no force acting on the state variable), and set the constraints on the control to be the constant functions $a \equiv-100$ and $b \equiv 100$.

Figure 2 shows the solutions of the corresponding MPEC. It admits a positively measured biactive set (black region in the middle row, left plot) where both the state variable $y$ and the slack variable $\xi$ vanish, and shows the singular structure of the dual variable $p_{h}^{\star}$ (middle row, right plot). The control $u_{h}^{\star}$ (top row, right plot) is obtained from (5.3a). The state $y_{h}^{\star}$ (top row, left plot) is in fact influenced by the obtained control $u_{h}^{\star}$ such that it is closer to the desired values $\left(y_{h}^{\star}(0.125,0.125) \approx 0.3, y_{h}^{\star}(0.125,0.5)=y_{h}^{\star}(0.5,0.125) \approx 0.04, y_{h}^{\star}(0.375,0.375) \approx 0.07\right)$. Note that since the upper control constraint is active, the desired value of 1 can not be reached at $w=(0.125,0.125)$.

The convergence history of the solver for the discretized C-stationarity system is documented in Figure 3. In the top row, the decrease of the residual pertinent to the C-stationarity conditions is plotted against the outer iteration steps (over all mesh refinement steps). After 15 iterations of the outer loop, i.e., when $\gamma$ reaches approximately a value of $10^{3}$, the residual is considered sufficiently small and the data is refined. Then, the next outer loop starts. On most AFEM levels, the parameter $\gamma$ does not have to be increased in order to reach the breaking tolerance of tolo $=10^{-5}$ for the residual, i.e. the outer loop has to be run only once.

In the bottom row, the number of Newton-steps of the inner loop is plotted against the outer iterations. The algorithm performs zero to seven inner iterations until the residual pertinent to the penalized stationarity conditions drops below the specified tolerance. 

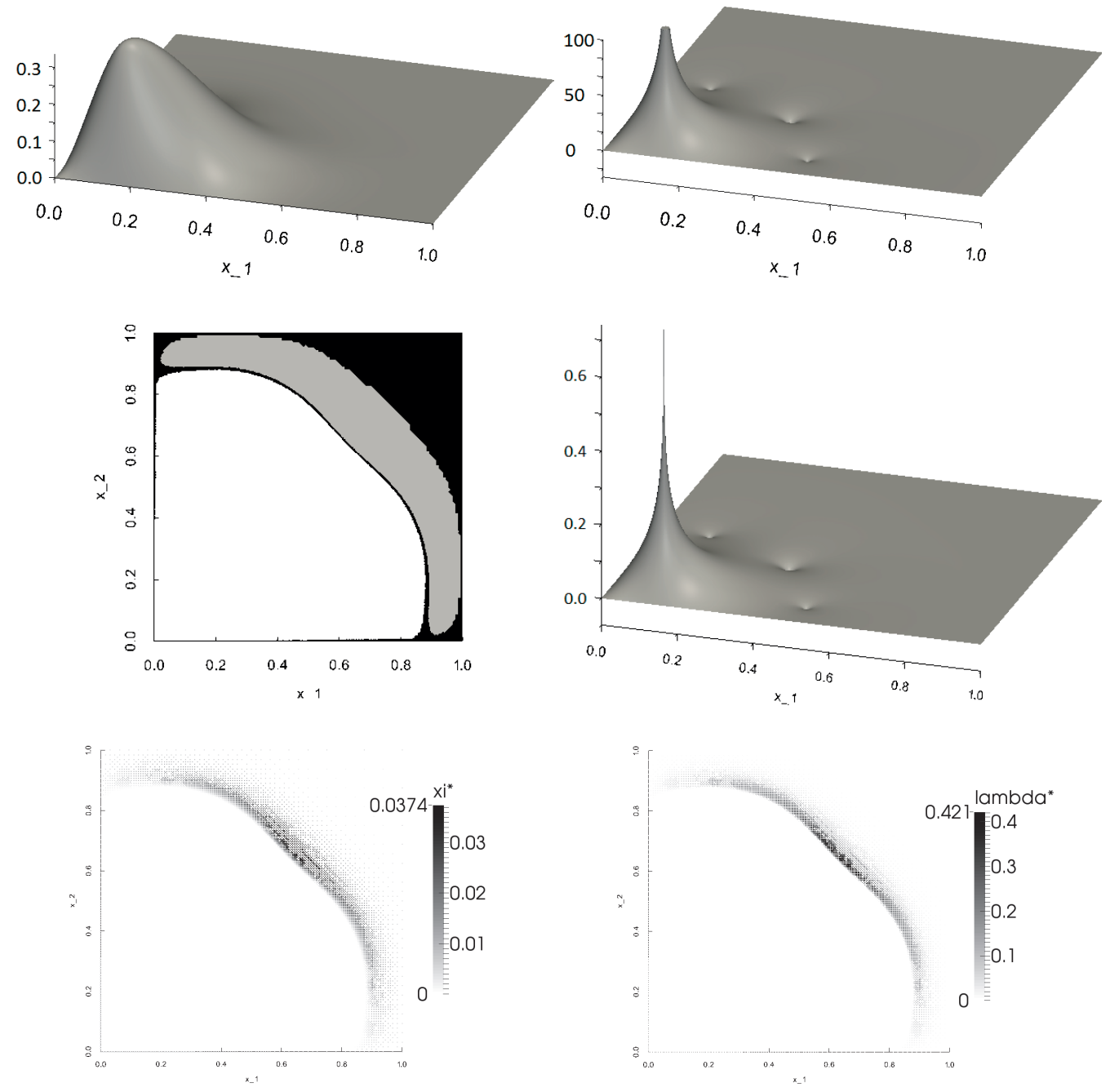

FIG. 2. Solution plots for Example 5.1. In the top row, $y_{h}^{\star}$ (left) and $u_{h}^{\star}$ (right) are shown. The middle row contains in the left plot the inactive set (white region), the biactive set (black region) and the strongly active set where $y_{h}^{\star}=0$ and $\xi_{h}^{\star}>0$ (grey region) as well as the adjoint state $p_{h}^{\star}$ in the right plot. The bottom row shows the values of the multipliers $\xi_{h}^{\star}$ (left) and $\lambda_{h}^{\star}$ (right). Nodes with values of 0 are not visible.

Figure 4 shows the adaptive mesh refinement process. The refinement takes place around the tracking points and at the boundary between active, inactive and biactive sets.

Finally we present our result on convergence of the adaptive method. Note that as we do not know the exact solution, we approximate it with a solution of the MPEC on the mesh that results from two additional uniform refinements of the finest adaptive grid. We denote the value of the objective at this approximated exact solution by $J^{\star}$, whereas $J^{U}$ and $J^{A}$ represent values of the objective in solutions on the uniform and on the adaptively refined meshes. Analogously we write 


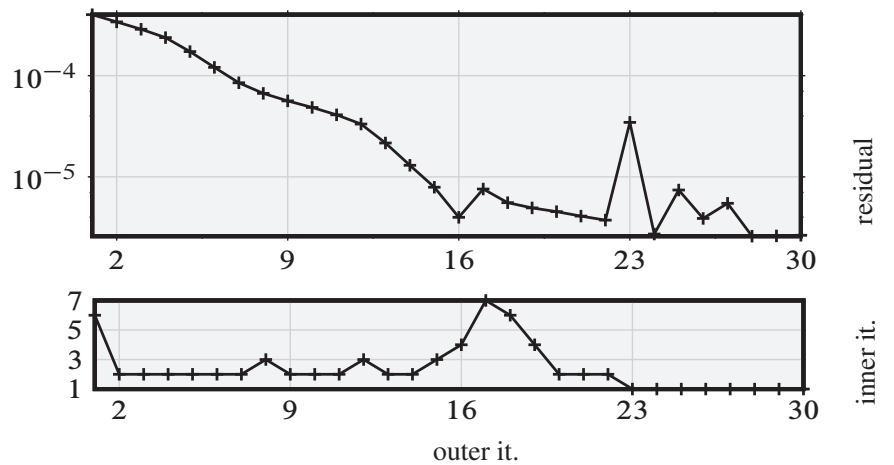

FIG. 3. Convergence history of the optimization routines for Example 5.1
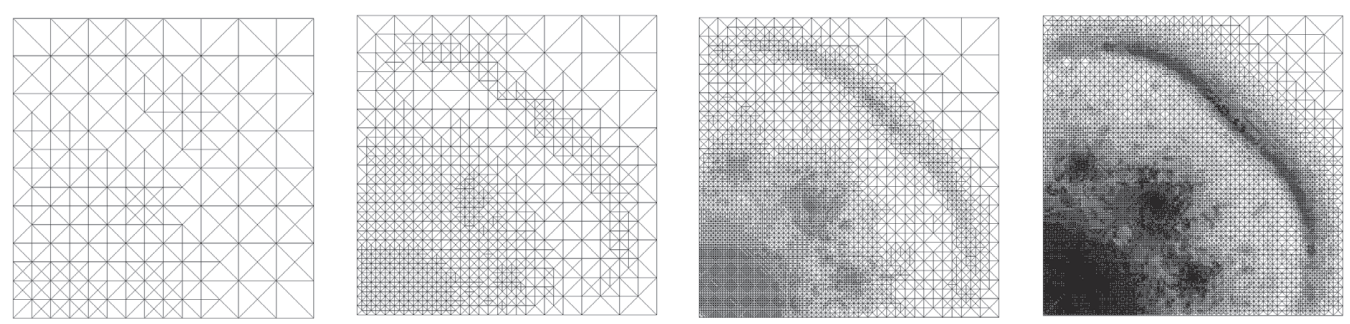

FIG. 4. Adaptively refined meshes at AFEM levels 3, 6, 9 and 12 for Example 5.1
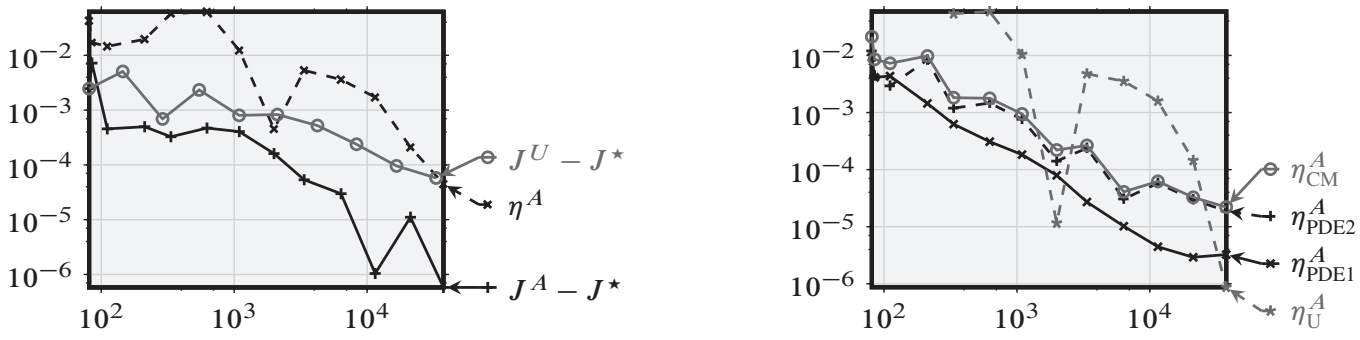

FIG. 5. Convergence of the full indicator $\eta^{A}$ and the errors $\left|J^{A}-J^{\star}\right|$ and $\left|J^{U}-J^{\star}\right|$ for adaptive and uniform refinement for Example 5.1

$\eta^{U}$ and $\eta^{A}$ for the total value of the indicator on uniform and adaptive meshes. Our error indicator shows good convergence properties:

The left plot in Figure 5 compares the error $\left|J^{A}-J^{\star}\right|$ on adaptively refined meshes (solid black line), the error $\left|J^{U}-J^{\star}\right|$ on uniform meshes (solid gray line), and the total value of the error indicator in the adaptive solution (dashed black line). This convergence plot shows reliability of the indicator, i.e., $\eta^{A} \geqslant\left|J^{A}-J^{\star}\right|$. In addition, the solutions on the adaptively refined grid exhibit lower errors compared to solutions on a uniform grid with the same number of degrees of 

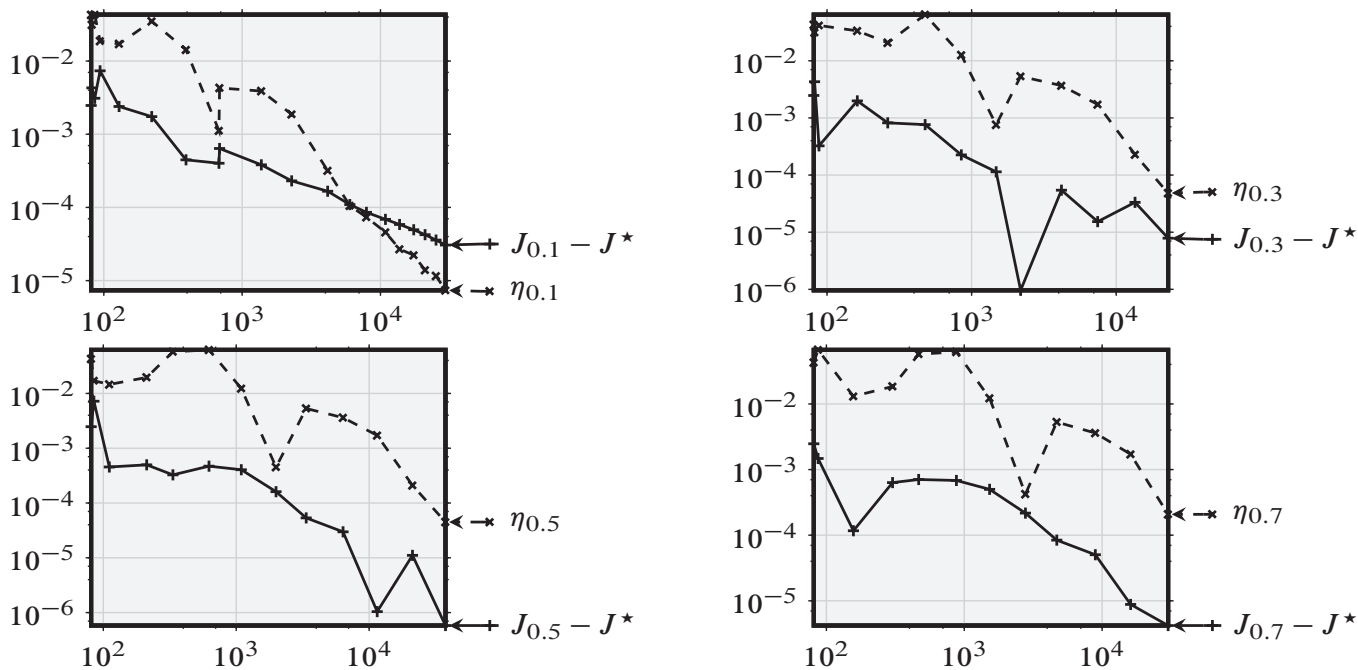

FIG. 6. Convergence comparison of the adaptive method for different marking parameters $\theta$ in Example 5.1

freedom. The right plot in Figure 5 shows the different components of the error indicator. Here, the part that represents the mismatch in complementarity with respect to the control constraints, namely $\eta_{\mathrm{U}}$ from (4.15), plays a crucial role in the AFEM convergence.

In Figure 6 we compare the behavior of the error indicator for different marking parameters $\theta$. From left to right and top to bottom, we set $\theta=0.1,0.3,0.5$ and 0.7 and write $J_{\theta}$ for the associated objective values. For small values of $\theta$ only a small number of triangles is in fact refined in each step. The plots in the top row show that this affects the convergence rate of the error in the objective and has an adverse impact on the reliability of the estimator. On the other hand, large values of $\theta$ result in a more uniform refinement, and thus again lead to an adverse effect on the convergence rate, compare the right plot in the bottom row.

EXAMPLE 5.2 The second example admits a less regular solution due to the non-convexity of the L-shaped domain $\Omega=(-1,0) \times(-1,1) \cup(-1,1) \times(0,1)$. The set of tracking points is now

$$
I=\{(-0.125,0.125),(-0.25,-0.25),(0,0.5),(-0.5,0),(0.25,0.25)(-0.375,0.375)\}
$$

and we want the state variable to take the value $y_{w}=1$ for $w=(-0.125,0.125)$ and the value 0 at the remaining tracking points. The parameter belonging to the control costs is again set to $v=0.003$, the force acting on the state is $f \equiv 0$ and the control constraints are removed.

Figure 7 shows the solutions of the corresponding MPEC. The problem is again non-smooth owing to its biactive set. Note that since there are no control constraints in this example, we achieve an even better point-tracking of the state $\left(y_{h}^{\star}(-0.125,0.125) \approx 0.6, y_{h}^{\star}(-0.25,-0.25)=\right.$ $\left.y_{h}^{\star}(0.25,0.25) \approx 0.06, y_{h}^{\star}(0,0.5)=y_{h}^{\star}(0.5,0) \approx 0.08, y_{h}^{\star}(-0.375,0.375) \approx 0.1\right)$.

The convergence history of the MPEC-solver is plotted in Figure 8. The structure is the same as in Example 5.1. Here, the outer loop takes 22 iterations before refining the mesh for the first time, and thus increases $\gamma$ to a value of approximately $10^{4}$. The penalized problem is solved in one to eight Newton iterations. 

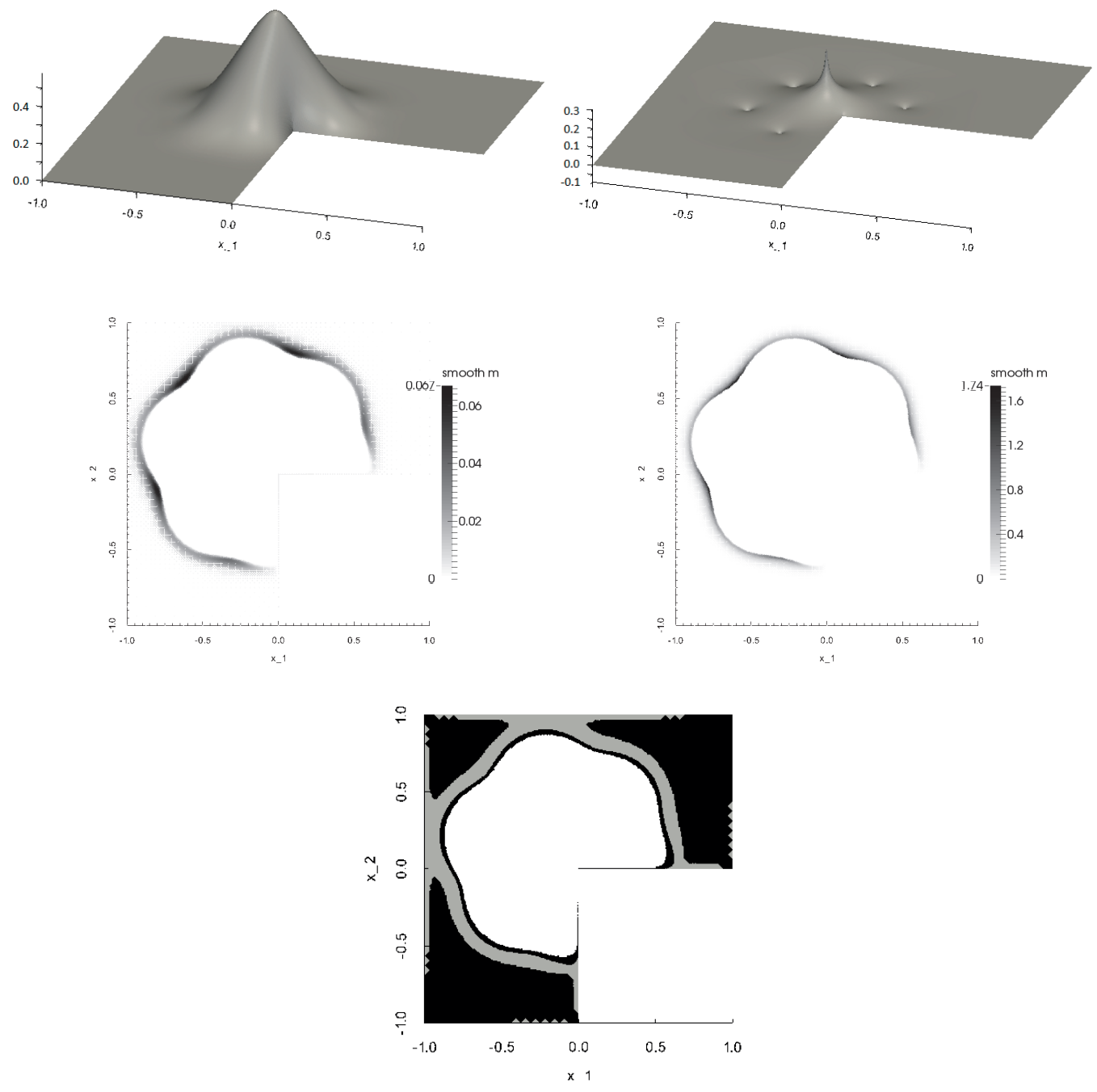

FIG. 7. Solution plots for Example 5.2. In the top row $y_{h}^{\star}$ (left) and $p_{h}^{\star}$ (right) are shown. There are no control constraints and thus $u_{h}^{\star}=\frac{1}{v} p_{h}^{\star}$. The middle row shows the values of the multipliers $\xi_{h}^{\star}$ (left) and $\lambda_{h}^{\star}$ (right). Nodes with values of 0 are not visible. The bottom row shows the inactive set (white region), the biactive set (black region) and the strongly active set where $y_{h}^{\star}=0$ and $\xi_{h}^{\star}>0$ (grey region).

We once again document the adaptive refinement process in Figure 9. The grid is refined around the tracking points, as well as at the boundary between active and inactive sets and near the re-entrant corner.

In the convergence plot for the uniform and adaptive refinement we use the same notation as in Example 5.1. The left hand side of Figure 10 again shows reliability of the indicator. Furthermore, on adaptively refined meshes, the method shows not only lower errors but also faster convergence. In the right plot, we show again the summands pertinent to the primal and dual partial differential 


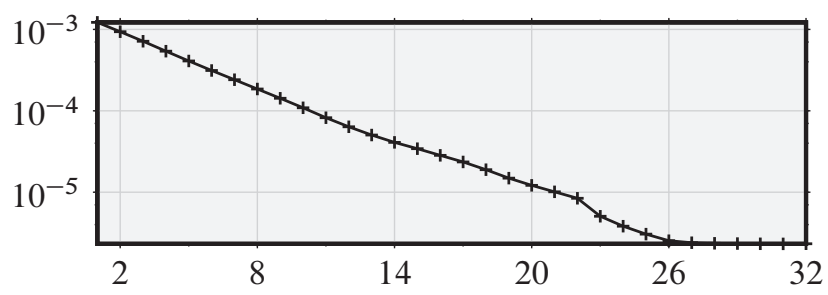

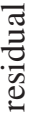

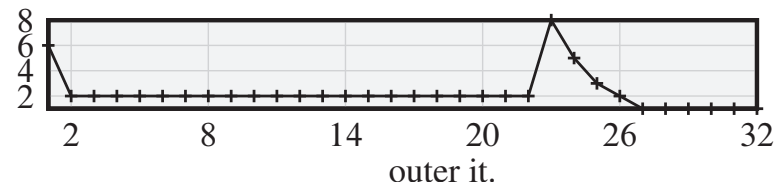

.ே.

FIG. 8. Convergence history of the optimization routines for Example 5.2
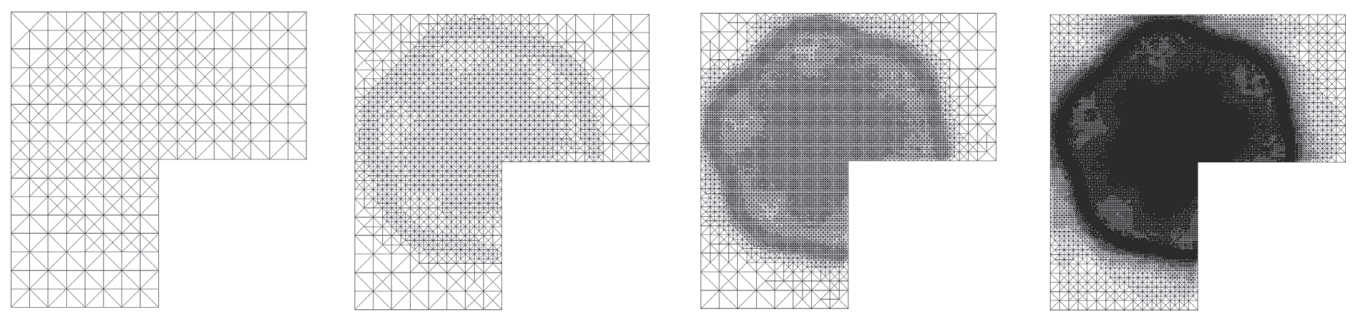

FIG. 9. Adaptively refined meshes at AFEM levels 2, 5, 8 and 11 for Example 5.2
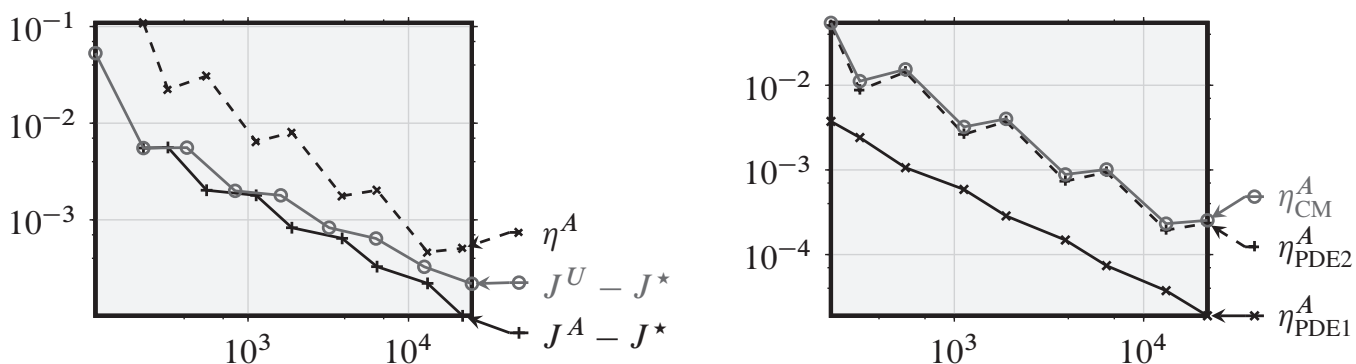

FIG. 10. Convergence of the full indicator $\eta^{A}$ and of the error $\left|J^{A}-J^{\star}\right|$ and $\left|J^{U}-J^{\star}\right|$ for adaptive and uniform refinement for Example 5.2

equations, $\eta_{\mathrm{PDE} 1}$ and $\eta_{\mathrm{PDE} 2}$, and the mismatch in complementarity of the state variable $y$, the slack variable $\xi$ and the multipliers $\lambda$ and $\mu, \eta_{\mathrm{CM}}$, separately. Note that since there are no constraints on the control $u$, the estimator $\eta_{\mathrm{U}}$ is zero. 
Acknowledgments. C.B. acknowledges support by the UK Engineering and Physical Sciences Research Council (EPSRC) Grant EP/H023364/1. M.H. and C.L. acknowledge support by the German Research Fund (DFG) through the Research Center MATHEON Project C28 and the SPP 1253 "Optimization with Partial Differential Equations", and the Austrian Science Fund (FWF) through the START Project Y 305-N18 "Interfaces and Free Boundaries" and by the Einstein Center of Mathematics Berlin. The authors wish to thank Andreas Dedner for his valuable contribution to this work.

\section{REFERENCES}

1. ACHDOU, Y., An inverse problem for a parabolic variational inequality arising in volatility calibration with american options, SIAM J. Control Optim. 43 (2005), 1583-1615. Zbl1075. 35100 MR2137495

2. AtKinson, K., \& Han, W., Theoretical Numerical Analysis: A Functional Analysis Framework, Springer, 2009. Zbl1181.47078 MR2511061

3. Babuška, I., Whiteman, J., \& Strouboulis, T., Finite Elements: An Introduction to the Method and Error Estimation, Oxford University Press, 2011. Zbl1206.65246 MR2857237

4. Bangerth, W., \& Rannacher, R., Adaptive Finite Element Methods for Differential Equations, Birkhäuser Verlag, Basel, 2003. Zbl1020.65058 MR1960405

5. B ̈̈NSCH, E., Local mesh refinement in 2 and 3 dimensions, IMPACT of Computing in Science and Engineering 3 (1991), 181-198. Zb10744.65074 MR1141298

6. BARbu, V., Optimal Control of Variational Inequalities, Addison-Wesley Educational Publishers Inc, 1984. Zb10574. 49005 MR0742624

7. Bastian, P., Blatt, M., Dedner, A., Engwer, C., Fahlke, J., Gräser, C., Klöfkorn, R., Nolte, M., Ohlberger, M., \& SAnder, O., DUNE web page, 2011. http://www.dune-project. org.

8. Bastian, P., Blatt, M., Dedner, A., Engwer, C., Klöfkorn, R., Kornhuber, R., OHLBERGER, M., \& SANDER, O., A generic grid interface for parallel and adaptive scientific computing. Part II: Implementation and tests in DUNE, Computing 82 (2008), 121-138. Zbl1151. 65088 MR2421580

9. Bastian, P., Blatt, M., Dedner, A., Engwer, C., Klöfkorn, R., Ohlberger, M., \& Sander, O., A generic grid interface for parallel and adaptive scientific computing. Part I: Abstract framework, Computing 82 2-3 (2008), 103-119. Zbl1151. 65089 MR2421579

10. Becker, R., KAPp, H., \& RANnACher, R., Adaptive finite element methods for optimal control of partial differential equations: Basic concept, SIAM J. Control Optim. (2000). Zb10967.65080 MR1780911

11. Benedix, O., \& VeXler, B., A posteriori error estimation and adaptivity for elliptic optimal control problems with state constraints, Comput. Optim. Appl. 44 (2009), 3-25. Zbl1192. 49031 MR2556843

12. Blatt, M., \& Bastian, P., The iterative solver template library, Applied Parallel Computing. State of the Art in Scientific Computing (2007), B. Kågström, E. Elmroth, J. Dongarra,, \& J. Waśniewski, Eds., vol. 4699 of Lecture Notes in Computer Science, Springer, 666-675.

13. Boccardo, L., An $L^{s}$-estimate for the gradient of solutions of some nonlinear unilateral problems, Ann. Mat. Pura Appl. 141 (1985), 277-287. Zbl0599. 49009 MR0816788

14. Bonnans, J. F., Cominetti, R., \& Shapiro, A., Sensitivity analysis of optimization problems under second order regular constraints, Math. Oper. Res. 23 (1998), 806-831. Zb10977. 90053 MR1662414

15. Brézis, H., \& Stampacchia, G., Sur la regularité de la solution d'inéquations elliptiques, Bulletin de la Société Mathématique de France 96 (1968), 153-180.

16. Burri, A., Dedner, A., Klöfkorn, R., \& Ohlberger, M., An efficient implementation of an adaptive and parallel grid in DUNE, Proc. of the 2nd Russian-German Advanced Research Workshop on Computational Science and High Performance Computing (2005). 
17. Dedner, A., Klöfkorn, R., Nolte, M., \& Ohlberger, M., A generic interface for parallel and adaptive scientific computing: Abstraction principles and the DUNE-FEM module, Computing 90 (2010), 165-196. MR2735465

18. Dedner, A., Klöfkorn, R., Nolte, M., \& Ohlberger, M., DUNE-FEM web page, 2011. http: //dune.mathematik.uni-freiburg.de.

19. Gaevskaya, A., Adaptive finite elements for optimally controlled elliptic variational inequalities of obstacle type, PhD thesis, Universität Augsburg, 2013.

20. Gaevskaya, A., Hoppe, R., \& Repin, S., Functional approach to a posteriori error estimation for elliptic optimal control problems with distributed control, Journal of Mathematical Sciences 144 (2007), 4535-4547. Zbl1202.65148 MR2584380

21. Glowinski, R., Lions, J.-L., \& Trémolières, R., Numerical Analysis of Variational Inequalities, vol. 8 of Studies in Mathematics and its Applications, North-Holland, Amsterdam, 1981. Zb10463.65046 MR0635927

22. GRÖGER, K., A $W^{1, p}$-estimate for solutions to mixed boundary value problems for second order elliptic differential equations, Math. Ann. 283 (1989), 679-687. Zb10646.35024 Mr0990595

23. GÜNTHer, A., \& HinZE, M., A posteriori error control of a state constrained elliptic control problem, J. Numer. Math. 16 (2008), 307-322. Zbl1161.65049 MR2493170

24. Hintermüller, M., \& Hoppe, R., Goal-oriented adaptivity in control constrained optimal control of partial differential equations, SIAM Journal on Control and Optimization 47 (2008), 1721-1743. Zbl1167.49029 MR2421327

25. Hintermüller, M., \& Hoppe, R., Goal-oriented adaptivity in pointwise state constrained optimal control of partial differential equations, SIAM Journal on Control and Optimization 48 (2010), 54685487. Zbl1208. 49025 MR2745781

26. Hintermüller, M., \& Hoppe, R., Goal-oriented mesh adaptivity for mixed control-state constrained elliptic optimal control problems, Applied and Numerical Partial Differential Equations, W. Fitzgibbon, Y. Kuznetsov, P. Neittaanmäki,, \& J. Périaux, Eds., vol. 15 of Computational Methods in Applied Sciences. Springer, Berlin-Heidelberg-New York, 2010, 97-111. Zbl1186.65080 MR2642683

27. Hintermüller, M., Hoppe, R., \& LÖBhard, C., A dual-weighted residual approach to goal-oriented adaptivity for optimal control of elliptic variational inequalities, ESAIM: COCV 20 (2014), 524-546. Zbl1287.49030 MR3264215

28. Hintermüller, M., \& KopackA, I., Mathematical programs with complementarity constraints in function space: C- and strong stationarity and a path-following algorithm, SIAM Journal on Optimization 20 (2009), 868-902. Zbl1189.49032 MR2515801

29. Hintermüller, M., \& KopacKA, I., A smooth penalty approach and a nonlinear multigrid algorithm for elliptic MPECs, Computational Optimization and Applications 50 (2011), 111-145. 10.1007/s10589009-9307-9. Zbl1229.49032 MR2822818

30. Hintermüller, M., Hoppe, R., Iliash, Y., \& Kieweg, M., An a posteriori error analysis of adaptive finite element methods for distributed elliptic control problems with control constraints, ESAIM: Control, Optimisation and Calculus of Variations 14 (2008), 540-560. Zbl1157.65039 MR2434065

31. Hoppe, R., Iliash, Y., IYyunni, C., \& SWeilam, N., A posteriori error estimates for adaptive finite element discretizations of boundary control problems, J. Numer. Math. 14 (2006), 57-82. Zb11104. 65066 MR2229819

32. Hoppe, R., \& KIEWEG, M., Adaptive finite element methods for mixed control-state constrained optimal control problems for elliptic boundary value problems, Computational Optimization and Applications $\mathbf{4 6}$ (2010), 511-533. Zbl1208.49037 MR2653722

33. Hoppe, R., \& M. KIEWEG, M., A posteriori error estimation of finite element approximations of pointwise state constrained distributed control problems, J. Numer. Math. 17 (2009), 219-244. Zbl1178.65070 MR2573568 
34. Kinderlehrer, D., \& Stampacchia, G., A free boundary value problem in potential theory, Ann. Inst. Fourier 25 (1975), 323-344. Zb10303. 31003 MR0507997

35. Kinderlehrer, D., \& Stampacchia, G., An Introduction to Variational Inequalities and Their Applications, Academic Press, New York, 1980. Zb10457. 35001 MR0567696

36. LI, R., LIU, W., MA, H., \& TANG, T., Adaptive finite element approximation for distributed elliptic optimal control problems, SIAM J. Control Optim. 41 (2002), 1321-1349. Zbl1034. 49031 MR1971952

37. LIU, W., \& YAN, N., A posteriori error estimates for distributed convex optimal control problems, Advances in Computational Mathematics 15 (2001), 285-309. Zbl1008. 49024 MR1887737

38. Luo, Z., PAng, J., \& RAlPh, D., Mathematical Programs with Equilibrium Constraints, Cambridge University Press, 1996. MR1419501

39. Meyers, N., An $L^{p}$-estimate for the gradient of solutions of second order elliptic divergence equations, Ann. Sc. Norm. Super. Pisa, Sci. Fis. Mat. III Ser. 17 (1963), 189-206. Zb10127. 31904 Mr0159110

40. Mignot, F., Contrôle dans les inéquations variationelles elliptiques, Journal of Functional Analysis 22 (1976), 130-185. Zb10364.49003 MR0423155

41. Neittaanmäki, P., Sprekels, J., \& Tiba, D., Optimization of Elliptic Systems: Theory and Applications, Springer Monographs in Mathematics. Springer, 2006. Zb11106.49002 MR2183776

42. Outrata, J., JARUŠEK, J., \& StARÁ, J., On optimality conditions in control of elliptic variational inequalities, Set-Valued and Variational Analysis 191 (2011), 23-42. Zbl1211.49036 MR2770895

43. Outrata, J., Kočvara, M., \& Zowe, J., Nonsmooth Approach to Optimization Problems with Equilibrium Constraints: Theory, Applications, and Numerical Results, vol. 28 of Nonconvex optimization and its applications, Kluwer Academic Publishers, 1998. Zb10947.90093 MR1641213

44. RePIn, S. A Posteriori Estimates for Partial Differential Equations, Radon Series on Computational and Applied Mathematics. De Gruyter, 2008. Zbl1162.65001 MR2458008

45. Rodrigues, J.-F., Obstacle Problems in Mathematical Physics, North-Holland, Amsterdam, 1987. Zb10606.73017 MR0880369

46. Rösch, A., \& Wachsmuth, D., A-posteriori error estimates for optimal control problems with state and control constraints, Numer. Math. 120 (2012), 733-762. Zbl1247. 65087 MR2892950

47. Scheel, H., \& Scholtes, S., Mathematical programs with complementarity constraints: Stationarity, optimality, and sensitivity, Math. Oper. Res. 251 (2000), 1-22. Zbl1073. 90557 MR1854317

48. Schiela, A., \& Wachsmuth, D., Convergence analysis of smoothing methods for optimal control of stationary variational inequalities with control constraints, ESAIM: Mathematical Modelling and Numerical Analysis 47 (2013), 771-787. Zbl1266.65112 MR3056408

49. VERFÜRTH, R., A review of a posteriori error estimation and adaptive mesh-refinement techniques, Wiley-Teubner, 1996. Zb10853.65108

50. Vexler, B., \& Wollner, W., Adaptive finite elements for elliptic optimization problems with control constraints, SIAM Journal on Control and Optimization 47 (2008), 509-534. Zbl1169. 65068 MR2373479

51. Zowe, J., \& Kurcyusz, S., Regularity and stability for the mathematical programming problem in Banach spaces, Appl. Math. Optim. 5 (1979), 49-62. Zb10401.90104 Mr0526427 